\title{
OBSCURED STAR FORMATION IN INTERMEDIATE-DENSITY ENVIRONMENTS: A SPITZER STUDY OF THE ABELL 901/902 SUPERCLUSTER
}

\author{
Anna Gallazzi ${ }^{1}$, Eric F. Bell ${ }^{1}$, Christian Wolf ${ }^{2}$, Meghan E. Gray ${ }^{3}$, Casey Papovich ${ }^{4}$, Marco Barden $^{5}$, Chien Y. \\ Peng $^{6}$, Klaus Meisenheimer ${ }^{1}$, Catherine Heymans ${ }^{7}$, Eelco van Kampen ${ }^{5}$, Rachel Gilmour ${ }^{8}$, Michael Balogh ${ }^{9}$, Daniel \\ H. McIntosh ${ }^{10}$, David BaCoN ${ }^{11}$, Fabio D. Barazza ${ }^{12}$, Asmus BöHM ${ }^{13}$, John A.R. Caldwell ${ }^{14}$, Boris HäUsSleR ${ }^{3}$, KNud \\ Jahnke $^{1}$, Shardha Jogee ${ }^{15}$, Kyle Lane ${ }^{3}$, Aday R. Robaina ${ }^{1}$, Sebastian F. Sanchez ${ }^{16}$, Andy Taylor ${ }^{17}$, Lutz Wisotzki ${ }^{12}$, \\ AND XIANZHONG ZHENG ${ }^{18}$ \\ ${ }^{1}$ Max-Planck-Institut für Astronomie, Königstuhl 17, D-69117 Heidelberg, Germany; gallazzi@mpia.de \\ ${ }^{2}$ Department of Physics, Denys Wilkinson Bldg., University of Oxford, Keble Road, Oxford, OX1 3RH, UK \\ ${ }^{3}$ School of Physics and Astronomy, University of Nottingham, Nottingham NG7 2RD, UK \\ ${ }^{4}$ Department of Physics Texas A \& M University College Station, TX 77843, USA \\ ${ }^{5}$ Institute for Astro- and Particle Physics, University of Innsbruck, Technikerstr. 25/8, A-6020 Innsbruck, Austria \\ ${ }^{6}$ NRC Herzberg Institute of Astrophysics, 5071 West Saanich Road, Victoria V9E 2E7, Canada \\ ${ }^{7}$ Department of Physics and Astronomy, University of British Columbia, 6224 Agricultural Road, Vancouver V6T 1Z1, Canada \\ ${ }^{8}$ European Southern Observatory, Alonso de Cordova 3107, Vitacura, Casilla 19001, Santiago 19, Chile \\ 9 Department of Physics and Astronomy, University of Waterloo, Waterloo, Ontario N2L 3G1, Canada \\ ${ }^{10}$ Department of Astronomy, University of Massachusetts, 710 North Pleasant Street, Amherst, MA 01003, USA \\ ${ }^{11}$ Institute of Cosmology and Gravitation, University of Portsmouth, Hampshire Terrace, Portsmouth PO1 2EG, UK \\ ${ }^{12}$ Laboratoire d'Astrophysique, École Polytechnique Fédérale de Lausanne (EPFL), Observatoire, CH-1290 Sauverny, Switzerland \\ 13 Astrophysikalisches Institut Potsdam, An der Sternwarte 16, D-14482 Potsdam, Germany \\ ${ }^{14}$ University of Texas, McDonald Observatory, Fort Davis, TX 79734, USA \\ ${ }^{15}$ Department of Astronomy, University of Texas at Austin, 1 University Station, C1400 Austin, TX 78712-0259, USA \\ ${ }^{16}$ Centro Hispano Aleman de Calar Alto, C/Jesus Durban Remon 2-2, E-04004 Almeria, Spain \\ ${ }^{17}$ The Scottish Universities Physics Alliance (SUPA), Institute for Astronomy, University of Edinburgh, Blackford Hill, Edinburgh, EH9 3HJ, UK \\ ${ }^{18}$ Purple Mountain Observatory, Chinese Academy of Sciences, Nanjing 210008, China \\ Received 2008 June 20; accepted 2008 September 19; published 2008 December 30
}

\begin{abstract}
We explore the amount of obscured star formation as a function of environment in the Abell 901/902 (A901/902) supercluster at $z=0.165$ in conjunction with a field sample drawn from the A901 and CDFS fields, imaged with the Hubble Space Telescope as part of the Space Telescope A901/902 Galaxy Evolution Survey and Galaxy Evolution from Morphology and Spectral Energy Distributions (SEDs) Survey. We combine the COMBO-17 near-UV/optical SED with Spitzer $24 \mu \mathrm{m}$ photometry to estimate both the unobscured and obscured star formation in galaxies with $M_{*}>10^{10} M_{\odot}$. We find that the star formation activity in massive galaxies is suppressed in dense environments, in agreement with previous studies. Yet, nearly $40 \%$ of the star-forming (SF) galaxies have red optical colors at intermediate and high densities. These red systems are not starbursting; they have star formation rates (SFRs) per unit stellar mass similar to or lower than blue SF galaxies. More than half of the red SF galaxies have low infrared-to-ultraviolet (IR-to-UV) luminosity ratios, relatively high Sérsic indices, and they are equally abundant at all densities. They might be gradually quenching their star formation, possibly but not necessarily under the influence of gas-removing environmental processes. The other $\gtrsim 40 \%$ of the red SF galaxies have high IR-to-UV luminosity ratios, indicative of high dust obscuration. They have relatively high specific SFRs and are more abundant at intermediate densities. Our results indicate that while there is an overall suppression in the SF galaxy fraction with density, the small amount of star formation surviving the cluster environment is to a large extent obscured, suggesting that environmental interactions trigger a phase of obscured star formation, before complete quenching.
\end{abstract}

Key words: galaxies: evolution - galaxies: general - galaxies: stellar content

Online-only material: color figures

\section{INTRODUCTION}

Much observational evidence gathered so far has established that the environment in which galaxies live plays an important role in shaping their properties, such as their star formation activity, gas content, and morphology, in the sense that galaxies in regions of high galaxy density tend to have less ongoing star formation, less cold gas, and more bulge-dominated morphology (Oemler 1974; Dressler 1980; Lewis et al. 2002; Gavazzi et al. 2002; Gómez et al. 2003; Balogh et al. 2004b; Kauffmann et al. 2004; McIntosh et al. 2004; Baldry et al. 2006). Yet a real concern is that most star formation indicators used to date are based on optical properties and are susceptible to the effects of dust attenuation. Indeed a number of studies using mid-infrared (mid-IR) or radio-derived star formation rates (SFRs) have found evidence for some unexpectedly intense bursts of star formation in intermediate-density regions (e.g., Miller \& Owen 2002; Coia et al. 2005; Fadda et al. 2008). The object of this paper is to use wide-field photometric redshift data, deep Spitzer data, and wide-field Hubble Space Telescope (HST) imaging of the $z=0.165$ Abell 901/902 (A901/902) supercluster to explore the incidence of dust-obscured star formation for low-SFR galaxies: is dust-obscured star formation important even at low SFRs, and how does it vary with environment? 


\subsection{Environment, Star Formation, and Morphology}

Historically, the first clear evidence that environment influences galaxy properties is the observed predominance of early-type galaxies in low-redshift clusters with respect to the field, along with a paucity of late-type, emission-line galaxies (e.g., Morgan 1961; Dressler 1980). The so-called morphologydensity relation appears to be in place already at $z \sim 1$, but varies quantitatively with redshift: between $z \sim 0.5$ and the present, the fraction of late-type spirals in intermediate-density regions decreases in favor of the population of S0 galaxies (Dressler et al. 1997; Smith et al. 2005; Postman et al. 2005). This has suggested that spiral galaxies evolve into smooth and passive systems such as S0s as they enter the dense environment of galaxy clusters.

Connected to the morphology-density relation is the decrease of the average SFR, as derived from optical colors or emission lines, with increasing environmental density (e.g., Balogh et al. 1998; Gavazzi et al. 2002; Pimbblet et al. 2002; Gómez et al. 2003). Among the two, the relation between color (or stellar age) and environment appears to be the most fundamental one: at fixed color, morphology shows only a weak residual dependence on environment (Blanton et al. 2005; Wolf et al. 2007). Moreover, the link between the morphology-density and the SFR-density relations has significant scatter: not all spirals in clusters appear to be star-forming, at least on the basis of their optical spectra (Poggianti et al. 1999; Goto et al. 2003). The question remains whether these spirals are really passive or they have star formation activity that escapes detection in the optical. Indeed selection of passive spirals on the basis of their emission lines can be contaminated by dusty early-type spirals with low levels of star formation activity that could instead be detected using, e.g., mid-IR colors (Wilman et al. 2008).

The SFR-density relation extends to very low local galaxy number densities (e.g., Lewis et al. 2002; Gómez et al. 2003) and dark matter densities (Gray et al. 2004), corresponding to the outskirts of clusters and the densities of groups. This suggests that not only the cores of clusters impact galaxy properties but galaxies may experience significant preprocessing in systems with lower density and lower velocity dispersion such as groups, before entering the denser and hotter environment of the cluster (e.g., Zabludoff 2002; Fujita 2004).

\subsection{Environmental Physical Processes}

Several processes can act on galaxies as they interact with their surrounding environment. The intensity and timescale of individual processes may also vary with galaxy mass and during the galaxy lifetime as it moves through different density environments (for a review, see Boselli \& Gavazzi 2006). The gas content and hence the star formation activity of galaxies can be affected by interaction with the intracluster medium (ICM). The cold gas reservoir can be stripped due to the rampressure experienced by galaxies falling at high velocities in the dense ICM of the cluster (Gunn \& Gott 1972; Quilis et al. 2000). Ram-pressure stripping can lead to fast truncation of star formation and its action can be recognized from truncated $\mathrm{H} \alpha$ profiles (Koopmann \& Kenney 2004), asymmetrical gas distribution and deficiency in the cold H I gas (Giovanelli \& Haynes 1985; Cayatte et al. 1990; Solanes et al. 2001) of many spiral galaxies in local clusters. It is possible that on the front of compression of the cold gas due to ram-pressure a burst of star formation is induced (e.g., Gavazzi \& Jaffe 1985; Gavazzi et al. 2003). Another gas-stripping process, that affects star formation on longer timescales (of few Gyr) than ram-pressure, is the socalled "strangulation" or "starvation"; assuming that galaxies are surrounded by a halo of hot diffuse gas, this can be removed when galaxies become satellites of larger dark matter halos (Larson et al. 1980; Balogh \& Morris 2000). Star formation can continue consuming the cold disk gas, but will eventually die out for the lack of supply of new fresh gas.

The gas distribution, star formation activity, and morphology of galaxies can be altered also via interaction with other galaxies. Mergers between two equally massive gas-rich galaxies can lead to the formation of a spheroidal system (e.g., Toomre \& Toomre 1972; Barnes 1988; Kauffmann et al. 1993). The merger can trigger an intense burst of star formation (e.g., Kennicutt et al. 1987), rapidly consuming the cold gas, and then exhausting due to feedback processes (Springel et al. 2005). Merging and slow galaxy-galaxy encounters are favored in groups and in the infall region of clusters (e.g., Moss 2006). At higher densities, galaxies can be affected by the cumulative effect of several rapid encounters with other cluster members, a mechanism known as "galaxy harassment" (Moore et al. 1998). After a transient burst of star formation, galaxy harassment leads to substantial change in morphology. This mechanism can start to operate at intermediate densities, inducing density fluctuations in the gas (Porter et al. 2008).

\subsection{Dust-Obscured Star Formation in Dense Environments?}

The net effect of the various mechanisms of interaction of galaxies with environment is an accelerated depletion or exhaustion of the gas reservoir and hence a suppression of the star formation activity. Many of these mechanisms, however, can lead to a temporary enhancement of star formation, either due to gas compression (e.g., ram pressure) or density fluctuations that funnel the gas toward the center triggering nuclear activity (e.g., tidal interactions). The gas and dust column density is likely to increase during such processes and star formation can be to a large extent obscured and escape optical detection. Star formation indicators that are not affected by dust attenuation need to be adopted in order to quantify the occurrence of these obscured star formation episodes.

Already several studies based on observations in the thermal IR or in the radio have identified significant populations of IR-bright or radio-bright galaxies in the outer regions of nearby and intermediate redshift galaxy clusters (e.g., Smail et al. 1999; Miller \& Owen 2002, 2003; Best 2004; Coia et al. 2005). Miller \& Owen (2002) find that up to $20 \%$ of the galaxies in 20 nearby Abell clusters have centrally concentrated dust-obscured star formation. These galaxies have different spatial distribution with respect to normal star-forming (SF) galaxies or active galactic nuclei (AGNs); they are preferentially found in intermediatedensity regions. In the A901/902 cluster at $z=0.165$, Wolf et al. (2005) have identified an excess of dusty red galaxies with young stellar populations in the intermediate density, infalling region of the cluster. Other studies have identified a population of red SF galaxies both in the field (Hammer et al. 1997) and in clusters (Verdugo et al. 2008). These galaxies could be mistakenly classified as post-starburst on the basis of their weak emission lines (Poggianti et al. 1999; Bekki et al. 2001). It is interesting to note that populations of red, IR-bright SF galaxies are often found in filaments (e.g., Fadda et al. 2000, 2008; Porter et al. 2008) and in unvirialized or merging clusters (e.g., Miller \& Owen 2003; Geach et al. 2006; Moran et al. 2007). Significant populations of starburst, IR-bright galaxies have also been found 
in a dynamically young cluster at $z=0.83$ by Marcillac et al. (2007). These systems could in fact be more abundant at higher redshift (Saintonge et al. 2008) as expected from the increase in cosmic star formation activity. Recently, Elbaz et al. (2007) have shown that the detection of these galaxies with the use of dust-independent SFR indicators can even lead to a reversal of the star-formation-density relation at $z \sim 1$.

In this work we want to explore as a function of local galaxy density the importance in the local universe of the star formation "hidden" among red galaxies, that would be missed by optical, dust-sensitive SFR indicators. Uniquely, we wish to push to modest SFRs $\left(\sim 0.2 M_{\odot} \mathrm{yr}^{-1}\right)$, in order to constrain the starformation mode of typical (not rare starbursting) systems. There are two key requirements for such a study: (1) obscurationfree SFR indicators, ideally given by the combination of deep thermal IR and UV, in order to obtain a complete census of the total (obscured and unobscured) SFR; (2) a long baseline in environmental density covering from the cluster cores to the field in order to quantitatively characterize the SFR-density relation. We analyze the COMBO-17 CDFS and A901 fields at $z<0.3$, complementing the UV/optical photometry from сомво-17 with Spitzer $24 \mu \mathrm{m}$ data and with $H S T V$-band imaging from the Galaxy Evolution from Morphology and Spectral Energy Distributions (GEMS) Survey and the Space Telescope A901/902 Galaxy Evolution Survey (STAGES). The A901 field is particularly interesting in that it contains the supercluster A901/902 at $z=0.165$, a complex system with four main substructures probably in the process of accreting or merging, where mechanisms altering the star formation and morphological properties of galaxies might be favored (e.g., Gray et al. 2002, 2004; Wolf et al. 2005, 2007; Heymans et al. 2008; Gray et al. 2008).

We present the sample and the data in Section 2.1 and describe the derivation of SFR and environmental density in Sections 2.2 and 2.3. After discussing the classification into SF and quiescent galaxies in Section 3.1, we explore the dependence on local galaxy density of the fraction of (obscured and unobscured) SF galaxies and their contribution to the total star formation activity as a function of environment (Section 3.2). The properties of red SF galaxies, such as their SFR, mass, morphology, and dust attenuation, are compared to those of unobscured SF galaxies in Section 3.3. We summarize and discuss our results in Section 4. Throughout the paper we assume a cosmology with $\Omega_{m}=0.3$, $\Omega_{\Lambda}=0.7$, and $H_{0}=70 \mathrm{kms}^{-1} \mathrm{Mpc}^{-1}$.

\section{THE DATA}

We describe here the sample analyzed and the data available. Based on this, we describe the measurement of derived parameters such as stellar mass, SFR, and environmental density.

\subsection{The Sample and the Data}

The sample analyzed is drawn from two southern fields, the extended Chandra Deep Field South and the A901 field, covered in optical by the сомво-17 Survey (Wolf et al. 2003) and at $24 \mu \mathrm{m}$ by MIPS onboard the Spitzer Space Telescope (Rieke et al. 2004). СомBo-17 has imaged three $34^{\prime} \times 33^{\prime}$ fields (CDFS, A901, S11) down to $R \sim 24$ in five broad and 12 medium bands sampling the optical SED from 3500 to $9300 \AA$. The 17passband photometry in conjunction with a library of galaxy, star, and AGN template spectra has allowed object classification and redshift assignment for $99 \%$ of the objects, with a redshift accuracy of typically $\delta z /(1+z) \sim 0.02$.
Spitzer has imaged at $24 \mu \mathrm{m}$ a field of $1 \mathrm{deg} \times 0.5 \mathrm{deg}$ around CDFS as part of the MIPS Guaranteed Time Observations (GTOs) and an equally sized field around the A901/902 supercluster (A901 field) as part of Spitzer GO-3294 (PI: E. F. Bell). The data have been acquired in a scan-map mode with individual exposures of $10 \mathrm{~s}$. In CDFS, the $24 \mu \mathrm{m}$ data reach a $5 \sigma$ depth of $83 \mu \mathrm{Jy}$ (see Papovich et al. 2004 for a technical description of source detection and photometry). In A901, the same exposure time reached a $5 \sigma$ depth of $97 \mu \mathrm{Jy}$, owing to the high contribution of zodiacal light at its near-ecliptic position. In what follows, we use both catalogs to $83 \mu \mathrm{Jy}$ ( $5 \sigma$ and $4 \sigma$ for CDFS and A901, respectively), noting that our conclusions are little affected if we adopt brighter limits for sample selection. The $24 \mu \mathrm{m}$ sources have been matched to galaxies with a photometric redshift estimate in the сомво-17 catalog, adopting a $1^{\prime \prime}$ matching radius. We omit sources within $4^{\prime}$ of the bright M8 Mira variable IRAS 09540-0946 to reduce contamination from spurious sources in the wings of its PSF.

The A901 сомво-17 field hosts the cluster complex A901/ 902 composed by the substructures A901a, A901b, A902, and the SW group at a redshift of $z=0.165$ within a projected area of $5 \times 5 \mathrm{Mpc}^{2} \mathrm{~h}_{70}^{-2}$. A quarter square degree field centered on the A901/902 supercluster has been imaged in the filter F606W with the HST Advanced Camera for Surveys (ACS) producing an 80 orbit mosaic, as part of STAGES (Gray et al. 2008). An area of 800 square arcminutes centered on the extended CDFS has also been imaged with HST/ACS in the F606W and F850LP filters, as part of the GEMS program (Rix et al. 2004). In the GEMS Survey object detection was carried out using the SExtractor software (Bertin \& Arnouts 1996) in a dual configuration that optimizes deblending and detection threshold (Caldwell et al. 2008). As described by Gray et al. (2008), a similar strategy for source detection has been adopted in STAGES. Both GEMS and STAGES imaging data have been processed using the pipeline GALAPAGOS (M. Barden et al. 2009, in preparation), which performs profile fitting and extract Sérsic indices (that we then use to morphologically characterize our sample) with the GALFIT fitting code (Peng et al. 2002).

X-ray data are also available for both the CDFS and the A901 field. X-ray data for the CDFS are available from the $\sim 1$ Ms Chandra point source catalog published by Alexander et al. (2003). The A901 field has been imaged by $X M M$ with a $90 \mathrm{ks}$ exposure and the catalog is presented by Gilmour et al. (2007). We use the X-ray information to identify possible AGN contribution among SF galaxies. To account for the different sensitivity of Chandra and XMM, we consider only sources with full band flux $>1.8 \times 10^{-15} \mathrm{erg} \mathrm{cm}^{-2} \mathrm{~s}^{-1}$, the faintest flux reached in the A901 field.

In this work we wish to study the dependence on environment of the star formation properties of low-redshift galaxies. To this purpose, we define a sample of galaxies in the redshift range $0.05<z<0.3$ from the CDFS and A901 fields (limited to the areas covered completely by Spitzer and сомво-17), down to an absolute magnitude of $M_{V}<-18$ (limited to those objects classified as galaxies by сомво-17). The sample peaks at an apparent magnitude of $m_{R} \sim 21$, covering the range $18 \lesssim m_{R} \lesssim 23$, with a (magnitude-dependent) redshift accuracy of $\sigma_{z} \lesssim 0.02$ for the majority of the galaxies, with a tail up to 0.05 (Wolf et al. 2004, 2005). The total sample comprises 1865 galaxies (1390 in the A901 field and 475 in the CDFS), of which 601 have a detection at $24 \mu \mathrm{m}$ above the $5 \sigma$ level. 
We will sometimes refer to "cluster" and "field" sample. The "cluster" sample is defined following Wolf et al. (2005), i.e., galaxies in the A901 field with redshift $0.155<z<0.185$, and it includes 647 galaxies. With this selection of the bright-end of the cluster population, the completeness reaches about the $92 \%$ level down to a magnitude of $R \sim 23$, but the contamination also rises to $40 \%$ (while it keeps below $20 \%$ for magnitudes brighter than $R=22$ ). The "field" sample is defined on the A901 field in the redshift ranges $0.05<z<0.125$ and $0.215<z<0.3$, and on the CDFS over the entire redshift range $0.05<z<0.3$, with a total of 981 galaxies.

\subsection{Stellar Mass and SFR}

Stellar mass estimates have been derived as outlined by Borch et al. (2006), using a set of template SEDs generated with the PÉGASE code, based on a library of three-component model starformation histories (SFHs), devised in such a way to reproduce the sequence of UV-optical template spectra collected by Kinney et al. (1996). The best-fitting SED, and hence the stellar massto-light ratio $\left(M_{*} / L\right)$, is obtained comparing the model colors with the observed ones. Stellar masses were derived adopting a Kroupa et al. (1993) initial mass function (IMF). Adopting a Kroupa (2001) or Chabrier (2003) IMF would yield differences in stellar mass of less than $10 \%$. Random errors amount to $\lesssim$ 0.3 dex on a galaxy by galaxy basis, while systematic uncertainties are typically of 0.1 dex for old stellar populations and up to 0.5 dex for galaxies with strong bursts (see also Bell et al. 2007).

The best indicator of the galaxy SFR combines the bolometric IR luminosity, assuming that it represents the bolometric luminosity of totally obscured young stars, and total UV luminosity or recombination lines such as $\mathrm{H} \alpha$ that trace instead the emission from unobscured young stars, thus giving a complete census of the luminosity emitted by young stars in a galaxy (e.g., Bell 2003; Calzetti et al. 2007). The IR data, combined with the near-UV-optical comBo-17 SED allows us to use such an SFR indicator. For this, we first need to estimate total UV and IR luminosities from monochromatic information.

To measure the total IR flux ideally we would need measurements at longer wavelengths (e.g., Helou et al. 1988; Dale $\&$ Helou 2002). We only have data in the $24 \mu \mathrm{m}$ MIPS passband which provides us with luminosities at rest-frame wavelength $\sim 23-18.5 \mu \mathrm{m}$ for the redshift interval $0.05-0.3$. The monochromatic 12-24 $\mu \mathrm{m}$ luminosity correlates well with the total IR luminosity, although it has some residual dependence on the gas metallicity (e.g., Papovich \& Bell 2002; Relaño et al. 2007; Calzetti et al. 2007). To convert the $24 \mu \mathrm{m}$ luminosity into total IR luminosity $(8-1000 \mu \mathrm{m})$, we use the Sbc template of the normal SF galaxy VCC 1987 from Devriendt et al. (1999). While there is certainly an intrinsic diversity in IR spectral shape at given luminosity or stellar mass, this results in a $\lesssim 0.3$ dex uncertainty in total IR luminosity, as inferred using the full range of Devriendt et al. (1999) templates.

The total UV luminosity (1216-3000 ^) is estimated from the luminosity $l_{\nu, 2800}$ in the COMBO- 17 synthetic band centered at $2800 \AA$ as $L_{\mathrm{UV}}=1.5 v l_{v, 2800}$. The rest-frame $2800 \AA$ band falls blueward of the observed сомво-17 $U$ band (centered at rest-frame $3650 \AA$ ) for galaxies at $z \lesssim 0.3$. The rest-frame luminosity at $2800 \AA$ thus requires an extrapolation of the bestfit model over about $200 \AA$ at the average redshift $z \sim 0.2$ of the sample. A factor of 1.5 in the definition of $L_{\mathrm{UV}}$ accounts for the UV spectral shape of a 100 Myr old stellar population with constant SFR (Bell et al. 2005).
We then translate UV and IR luminosities into SFR estimates following the calibration derived by Bell et al. (2005) from the PÉGASE stellar population synthesis code, assuming a $100 \mathrm{Myr}$ old stellar population and a Kroupa (2001) IMF:

$$
\operatorname{SFR}\left(M_{\odot} \mathrm{yr}^{-1}\right)=9.8 \times 10^{-11}\left(L_{\mathrm{IR}}+2.2 L_{\mathrm{UV}}\right) .
$$

This calibration has been derived by Bell et al. (2005) under the same assumptions adopted in the calibration of Kennicutt (1998); the two calibrations yield SFRs that agree within $\lesssim 30 \%$. A factor of 2.2 in front of the $L_{\mathrm{UV}}$ term in Equation (1) accounts for the light emitted by young stars redward of $3000 \AA$ and blueward of $1216 \AA$. We adopt Equation (1) to estimate the SFR for all galaxies detected at $24 \mu \mathrm{m}$. For galaxies which have upper limits to the $24 \mu \mathrm{m}$ flux, we omit the IR contribution and consider only the UV-optical emission. This is a rather conservative approach: the SFR of MIPS-undetected galaxies calculated in this way represents a lower limit to the true SFR. On the other hand, including the $L_{\mathrm{IR}}$ term calculated on the basis of the upper-limit flux of $83 \mu \mathrm{Jy}$ would overestimate the true SFRs of undetected galaxies.

We note that the adopted calibration relies on the assumption that the IR luminosity traces the emission from young stars only. There are few caveats to this assumption. Nuclear activity can also be responsible for at least part of the IR emission. X-ray data and optical identification of type-1 QSOs on both CDFS and A901 allow us to identify and exclude many AGNs from the sample, but we cannot exclude some contamination from obscured, Compton-thick AGNs. Risaliti et al. (1999) find that among local Seyfert 2 galaxies about $75 \%$ are heavily obscured (with hydrogen column densities $N_{H}>10^{23} \mathrm{~cm}^{-2}$ ) and $\sim 50 \%$ are Compton-thick $\left(N_{H}>10^{24} \mathrm{~cm}^{-2}\right)$. Among all $24 \mu \mathrm{m}$ detected galaxies in our sample only $\sim 3 \%$ are also $\mathrm{X}$-ray detected. Given the relatively faint limit reached in $\mathrm{X}$-ray $\left(L_{X} \gtrsim 10^{41} \mathrm{erg} \mathrm{cm}^{-2} \mathrm{~s}^{-1}\right)$, it is reasonable to assume that we potentially miss Compton-thick sources. Therefore, we expect only $\mathrm{a} \sim 3 \%$ contribution by Compton-thick AGNs. Moreover, the presence of an AGN does not necessarily imply that it dominates the total IR luminosity (Rowan-Robinson et al. 2005). Indeed A. R. Robaina et al. (2009, in preparation), based on Ramos Almeida et al. (2007) data and analysis, estimate that type-2 AGNs contribute only $\sim 26 \%$ of the total IR luminosity of their host galaxy.

In early-type galaxies circumstellar dust around red giant stars is expected to contribute to the mid-IR flux (see Temi et al. 2005, 2007 about the sensitivity of IR bands to different dust components in early-type galaxies). Nevertheless, the midIR in early-type galaxies can detect the presence of intermediateage stars and small amounts of ongoing star formation (Bressan et al. 2007; Young et al. 2008). As we discuss in Section 3.1, the majority of the early-type red-sequence galaxies are not detected at $24 \mu \mathrm{m}$. For those that are detected the SFR derived assuming that their IR luminosity traces young stellar populations is in any case not sufficient to classify them as SF galaxies.

There are some caveats also in the use of UV luminosity as tracer of young stars for early-type galaxies. While UV can help to detect recent episodes of low-level star formation, it can also be affected by evolved stellar populations (e.g., Rogers et al. 2007). These mainly contribute to the UV upturn at $1200 \AA$, i.e., at shorter wavelength than what we use, and therefore should not be a concern for the UV luminosities (and SFRs) derived in this work.

We thus believe that the caveats mentioned above do not appreciably affect the classification into SF and quiescent 


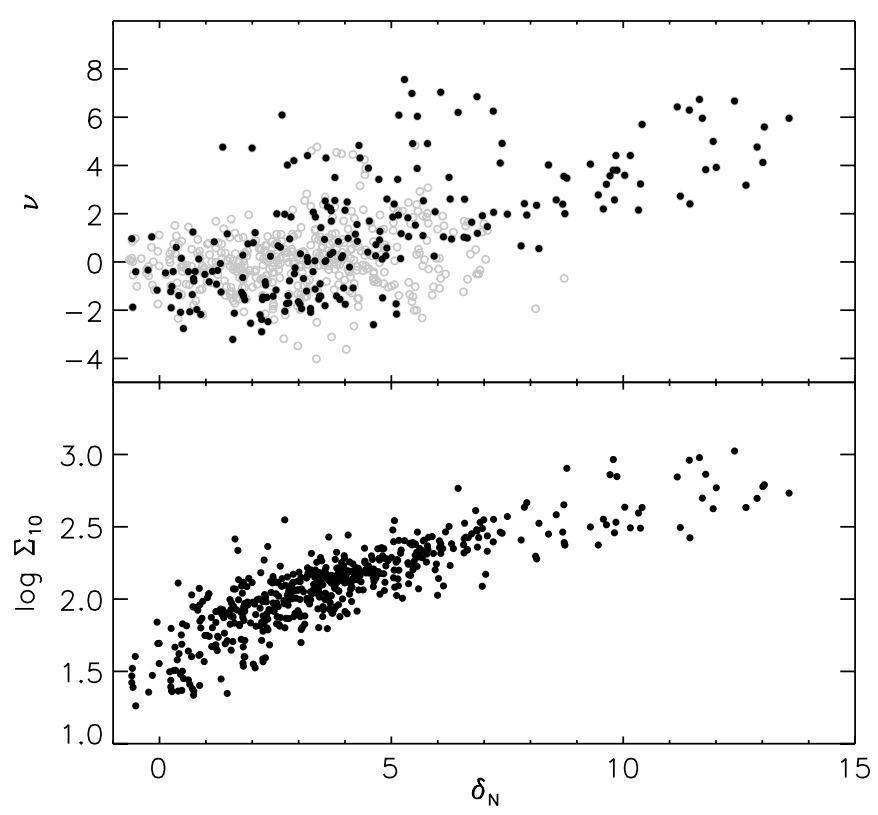

Figure 1. Local number overdensity $\delta_{N}$ used in this work is compared to the surface number density $\Sigma_{10}$ (lower panel) and to the surface mass density from weak lensing, as expressed by the parameter $v=\kappa / \sigma_{n}$ (upper panel). In the upper panel, empty gray circles indicate galaxies for which the dark matter density measure is not reliable because dominated by noise or systematics (see text). The comparison is performed only on galaxies in the A901/902 cluster.

galaxies used in this work (see Section 3.1) and our results. The combination of near-UV and deep $24 \mu \mathrm{m}$ data is indeed a powerful tool to detect unobscured and obscured star formation not only for starbursting galaxies but also in the regime of normal SF galaxies.

\subsection{Environmental Density}

The combination of the CDFS and of the A901 field, hosting the A901/902 supercluster, provides us with a large dynamic range of galaxy environments. As mentioned above, we have a well defined cluster sample, composed of galaxies within \pm 0.015 of the redshift of the cluster down to a magnitude of $M_{V}<-18$, and a comparison field sample. However, we wish to characterize the environmental galaxy density in a continuous way, such that it allows us to exploit the long baseline provided by the two fields.

We estimate the environmental density in a cylinder centered at the position of each galaxy in the sample, and express it in terms of overdensity with respect to an average redshiftdependent background density. The average background density, $\rho_{N}$, is calculated combining the three сомBO-17 fields, in redshift intervals of width 0.1 . In each redshift bin the total number of galaxies, including all objects classified as "galaxy" down to $R=23.5$ and correcting for completeness, ${ }^{19}$ is divided by the volume given by the total field area and the redshift depth. For each galaxy in the sample, the local density is obtained by counting the number $N_{\text {gal }}$ of galaxies (down to $R=23.5$, correcting for completeness), in a cylinder centered at the position of the galaxy of radius $0.25 \mathrm{Mpc}$ and depth given by the photometric redshift error for that galaxy $(\geqslant 0.015)$, and dividing by the volume $V$ of the cylinder corrected for edge effects. The local number density is then normalized to the average background density interpolated at the redshift of the galaxy. The

\footnotetext{
19 Galaxy completeness maps were estimated from simulations as a function of aperture magnitude, redshift, and $U-V$ color (see Wolf et al. 2004 for a detailed discussion).
}

local overdensity is then expressed as

$$
\delta_{N}=\frac{N_{\mathrm{gal}}}{V \rho_{N}}-1 .
$$

This estimate ranges from $\sim-1$ for very underdense regions, to 0 for average-density regions up to greater than 4 for the densities characteristic of the cluster.

Because of the relatively large errors associated with photometric redshifts (compared to spectroscopic ones) the galaxy density is effectively measured in volumes that extend $\gtrsim 80 \mathrm{Mpc}$ along the line of sight. In this respect, the local density adopted here represents a hybrid between projected density estimates (which neglect redshift information) and spectroscopic estimates (which smooth over much smaller scales of $\lesssim 8 \mathrm{Mpc}$ ). Thus, local densities calculated with photometric redshifts are biased toward the cosmic mean and suffer on a galaxy-bygalaxy basis from contamination from low-density interlopers in high-density regions (see Cooper et al. 2005 for a comparison of different density indicators). To quantify this effect, we have tested the density measures defined in Equation (2) against mock galaxy catalogs (containing superclusters similar to A901/902), applying the completeness of the COMBO-17 Survey. Using Equation (2), we have measured on the mock catalogs "observed" overdensities assuming realistic photometric redshift errors (those achieved with СОМво-17, allowing also for catastrophic errors), and "real" overdensities assuming the real observed redshift (including the peculiar velocity) and a redshift depth of $3 \times 10^{-3}$. The "observed" overdensities give a density ranking similar to the "real" overdensities, almost independently of galaxy luminosity and redshift. However, the magnitude of the "observed" overdensities is almost a factor of 10 lower than the "real" overdensities, owing to the difference in redshift path used to calculate the overdensity.

For galaxies in the A901/902 cluster, we could also compare our density estimates to other independent density estimators. Specifically, we compared with the projected galaxy density $\Sigma_{10}$ as defined by Wolf et al. (2007), which measures the number density of galaxies in an adaptive aperture of radius given by the average of the distance to the ninth and tenth nearest neighbor. The lower panel of Figure 1 shows a good correlation between $\Sigma_{10}$ and the galaxy overdensity $\delta_{N}$ measured in a fixed aperture.

The upper panel of Figure 1 compares $\delta_{N}$ with a measure of the total surface mass density from a weak lensing analysis of the HST STAGES data (Heymans et al. 2008). In this analysis, Heymans et al. (2008) present a pixelated map of the smoothed projected dark matter surface mass density $\kappa$ of the A901/902 cluster along with noise $\sigma_{n}$ and systematic error maps $B$, in order to assess the reliability of each feature. Following van Waerbeke (2000) we define a lensing density measure $v=\kappa / \sigma_{n}$ for the pixel region around each galaxy that corresponds to $\sim 20 \times 20 \mathrm{kpc}^{2}$. For $v \gg 1$ we can calculate a corresponding mass estimate, following Equation (4) in Heymans et al. (2008), where a galaxy with a lensing density measure $v=4$, for example, is enclosed in a local dark matter mass of $M(<20 \mathrm{kpc})=1 \times 10^{11} M_{\odot}$. For $v<1$ we enter a low to underdense regime, with the most negative regions showing the location of voids (Jain \& Van Waerbeke 2000; Miyazaki et al. 2002).

In this paper, we are particularly interested in the low- to intermediate-density regions of the A901/902 cluster. Unfortunately, for the weak lensing analysis, however, it is these lower density regions where systematic errors become important. We therefore introduce a selection criteria, following Heymans 

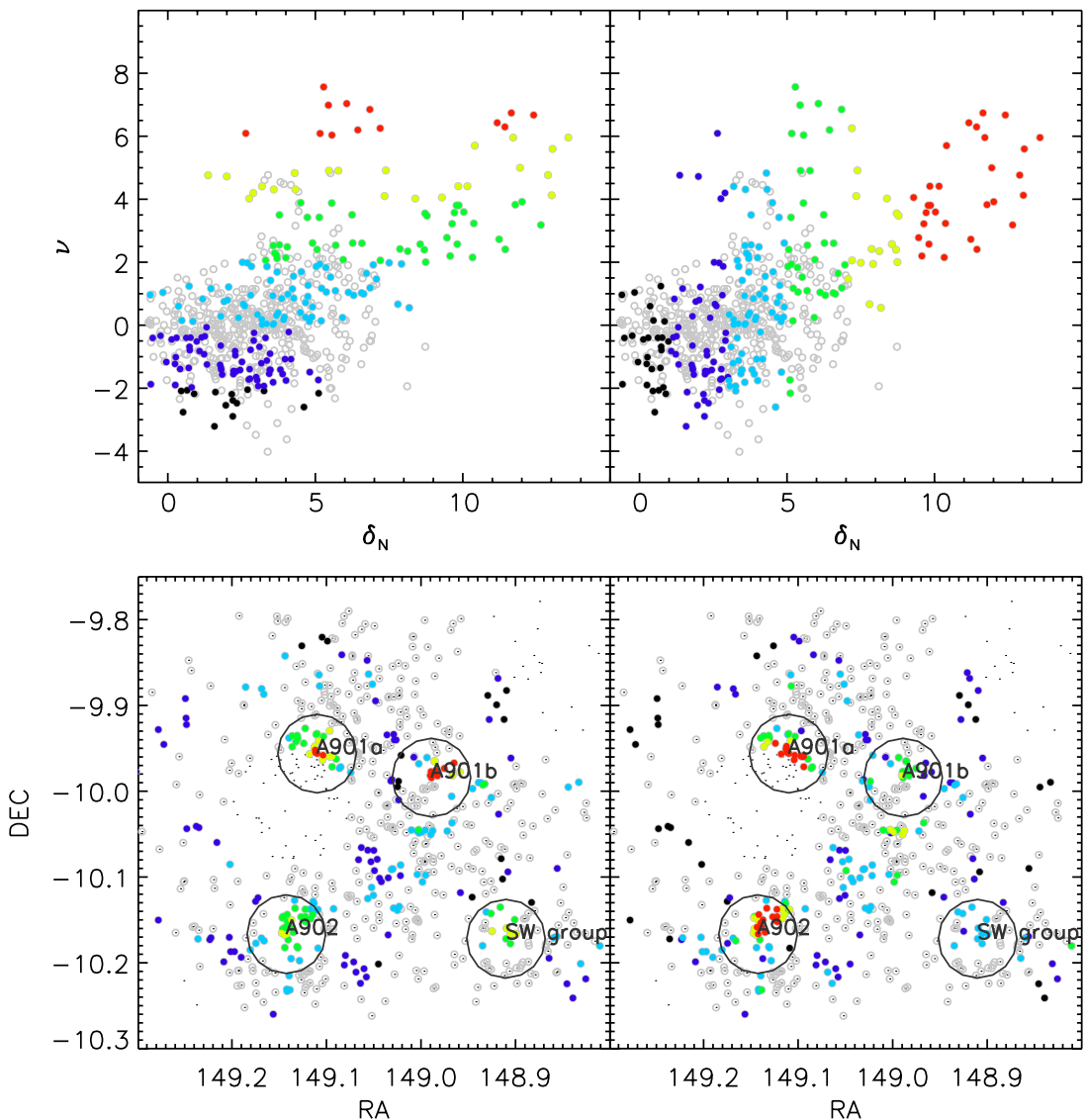

Figure 2. Lower panels: position on the sky of the A901/902 cluster galaxies, color-coded according to their environmental density, expressed either as dark matter density $v$ (left) or as galaxy number overdensity $\delta_{N}$ (right). The small dots refer to all comBo-17 $M_{V}<-18$ galaxies in the A901/902 cluster. The sample analyzed is limited to galaxies covered completely by comBO-17 and Spitzer. Gray empty circles are galaxies with an unreliable $v$ measures. The galaxies of interest in this comparison, i.e., those with reliable $v$ values, are represented with filled colored circles. Upper panels: relation between $v$ and $\delta_{N}$. The different colors indicate the density ranges in which galaxies are sorted in the corresponding lower panels.

et al. (2008), that the lensing density estimate $v$ is deemed reliable if the systematic error $B$ is either comparable to the noise $\sigma_{n}$ or less than half the amplitude of the signal $\kappa$. Figure 1 shows unreliable measures as open points. Comparing the reliable lensing density measurements $v$ (filled points) with $\delta_{N}$ shows a good correlation between these two environment variables. Taking only those galaxies with a reliable lensing measure, we show, in the lower left panel of Figure 2, the position in the sky of the A901/902 cluster galaxies, color-coded according to their $v$ value as indicated in the upper-left panel. The corresponding right panels refer to the galaxy overdensity $\delta_{N}$. We note in particular that the two dark matter peaks corresponding to the A901a and A902 cores are also identified as peaks in the galaxy distribution. Galaxies in these regions follow the main relation between $v$ and $\delta_{N}$ shown in the upper panels of Figure 1. The A901b core and SW group are instead associated with a lower galaxy density and show a larger spread to higher $v$ values at fixed $\delta_{N}$ that is not completely explained by larger errors on $v$.

While weak gravitational lensing techniques can provide a direct measure of the total matter density, this environment variable is integrated along the line of sight with contributions from mass at all redshifts. In the case of the A901/902 cluster, it is a reasonable approximation to place all the measured mass at the redshift of A901/902 as shown by Heymans et al. (2008) who find that the mass of this supercluster is significantly larger than the known galaxy groups and the CBI cluster behind A902 (Taylor et al. 2004). However, in the case of the CDFS field, mass is distributed fairly equally along the line of sight at relatively low density. It would therefore be very difficult to obtain a local matter density measure for this field from a weak lensing analysis even with the HST imaging that exists (see Heymans et al. 2005). For this reason we favor using $\delta_{N}$ as it permits local density measurements in both the field and cluster environments.

We note that the large redshift depth assumed in the density measure affects in particular the cluster sample, for which one would expect overdensities higher by about an order of magnitude. In what follows, however, we keep also for cluster galaxies the overdensities estimated over a depth set by the photometric redshift error, since we want to study cluster and field galaxies simultaneously with a consistent density measure. The distribution in density $\delta_{N}$ for the sample as a whole is shown in Figure 3. The dashed and dotted lines distinguish cluster galaxies from the field sample. As expected the field sample is concentrated in environments with density similar to or below the average background density. Cluster galaxies instead dominate at densities above $\delta_{N} \sim 2$.

\section{RESULTS}

We now describe the classification of galaxies on the basis of their SFR and optical color, that we will use throughout the paper (Section 3.1). Unless otherwise specified, the terms "red SF" and "obscured SF" used in the text refer to the same class of galaxies. We then investigate how the fraction of SF galaxies depends on galaxy environment, with particular attention to the extent of star formation "hidden" among red-sequence galaxies 


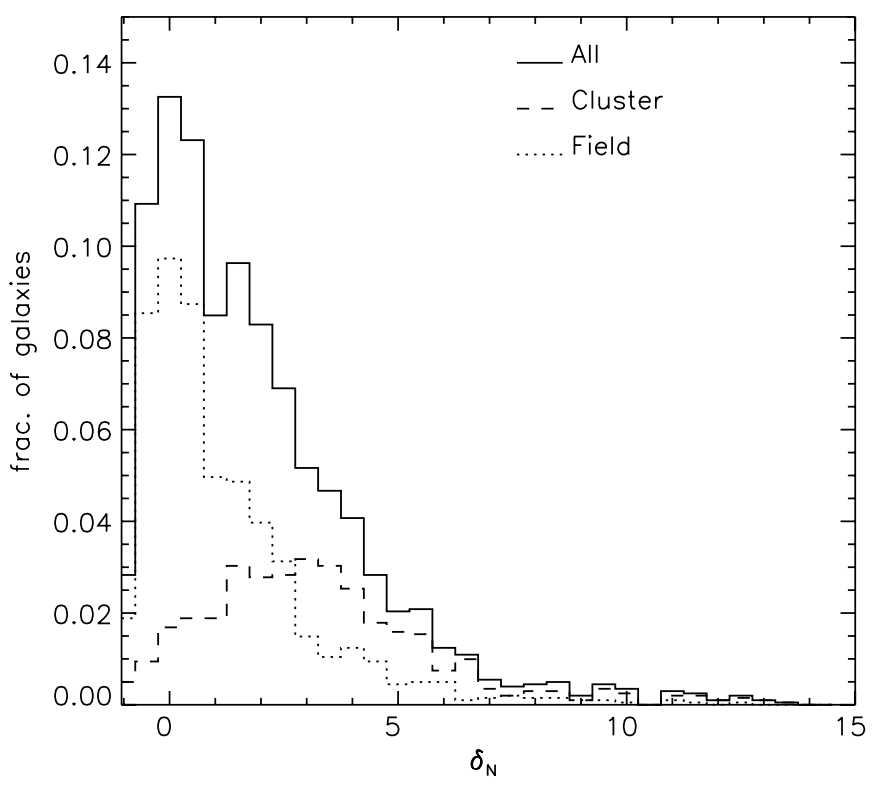

Figure 3. Distribution in local density $\delta_{N}$ for the sample as a whole (solid line), and then split into "cluster" (dashed line) and "field" galaxies (dotted line).

(Section 3.2). In Section 3.3 we analyze the star formation properties, morphology, and dust attenuation of red SF galaxies, as opposed to quiescent ellipticals and blue-cloud galaxies, as a function of environment.

\subsection{Galaxy Classes}

Figure 4 shows the distribution in the color-magnitude plane of galaxies in the A901/902 cluster (left panel) compared to galaxies in the field (right panel). The solid line indicates the magnitude-dependent color cut adopted to classify galaxies as red-sequence (redward of the line) or blue-cloud galaxies (blueward of the line). The cut is set $0.25 \mathrm{mag}$ blueward of the color-magnitude relation fitted by Bell et al. (2004) on the combined A901 + CDFS fields at $0.2<z<0.3$. Although the exact fraction of blue/red galaxies depends on the chosen color cut, it makes a little difference as long as the cut lies in the "gap" between the "blue" and the "red" peaks of the color distribution. Gray circles represent galaxies detected at $24 \mu \mathrm{m}$, with symbol size scaling according to their total IR luminosity. While we are not surprised to find a large number of $24 \mu \mathrm{m}$ emitting galaxies in the blue cloud, especially in the field, it is also noticeable a significant contamination of the cluster red sequence by IRluminous galaxies. A fraction of the IR luminosity may come from AGNs, although we note that only a small number of IRluminous red-sequence galaxies are identified as X-ray sources (large squares). We cannot exclude some contamination by obscured, Compton-thick AGNs, but we believe this is only a few percent (see Section 2.2). Figure 4 illustrates that $24 \mu \mathrm{m}$ information allows us to reveal a significant number of redsequence galaxies with IR luminosity in excess of $10^{10} L_{\odot}$, witnessing to a large extent ongoing star formation activity onto the red sequence, that would be otherwise undetected (or at least underestimated) because obscured by dust.

Before exploring the properties of red IR-luminous galaxies and their importance in terms of the total star formation budget as a function of environment, we define our classification into quiescent and SF galaxies, further distinguished into red and blue. We concentrate on galaxies more massive than $10^{10} M_{\odot}$, thus sampling the high-mass end of the mass function above which the red-sequence completeness is guaranteed up to redshift 0.3 (Borch et al. 2006). We set a threshold in specific $\mathrm{SFR}$ of $\log \left(\mathrm{SFR} / M_{*}\right)=-10.7$, which corresponds to a level of star formation of $0.2 M_{\odot} \mathrm{yr}^{-1}$ at the mass limit. We thus define galaxies as "quiescent" or "SF" depending on whether their specific SFR is below or above this level, respectively. We then separate "red SF" and "blue SF" galaxies according to the magnitude-dependent red-sequence cut shown in Figure 4.

The choice of the specific SFR limit is justified by the fact that the distribution of the massive galaxies in the sample in specific SFR (as measured in Equation (1)) is bimodal and the

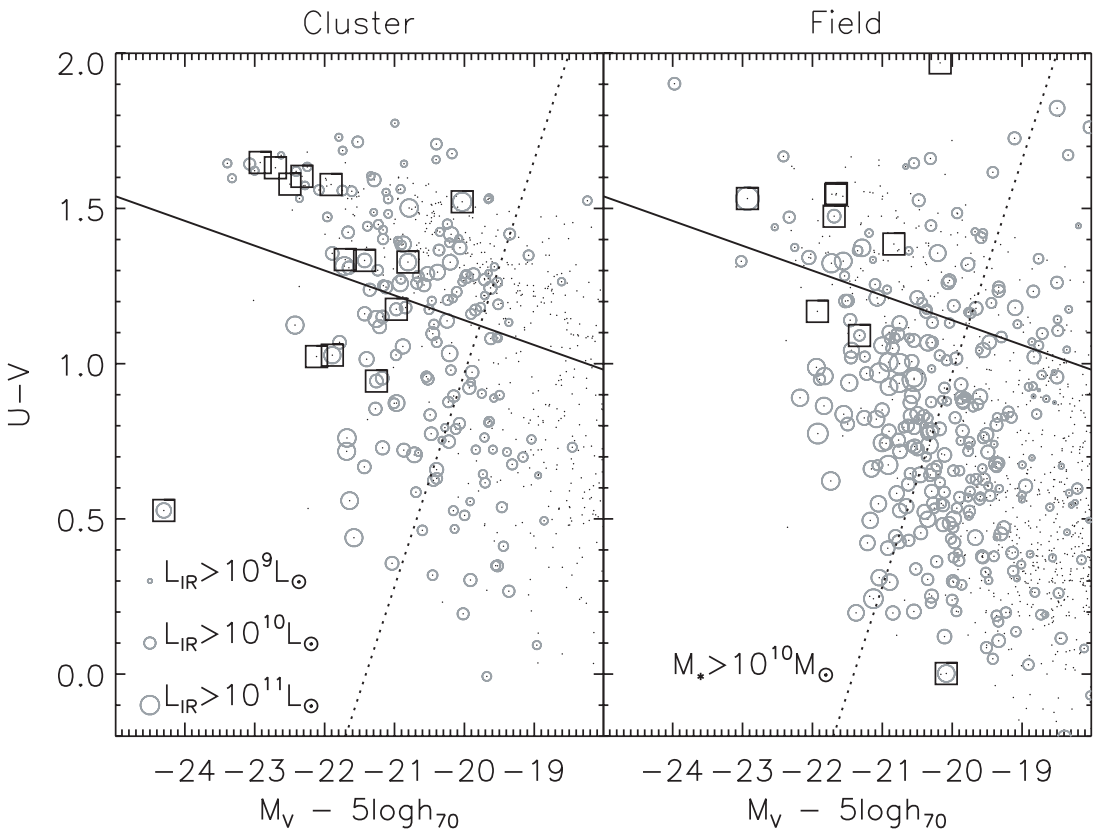

Figure 4. Rest-frame color-magnitude diagram for cluster galaxies (left) and for field galaxies (right). Gray circles represent galaxies detected at $24 \mu \mathrm{m}$, with symbol size scaling according to the total IR luminosity. Large squares indicate galaxies associated with X-ray sources. There is a significant fraction of $24 \mu \mathrm{m}$ emitting galaxies (with IR luminosity also in excess of $10^{10} L_{\odot}$ ) populating the cluster red sequence (solid line), in particular with masses greater than $10^{10} M_{\odot}($ dotted line). 


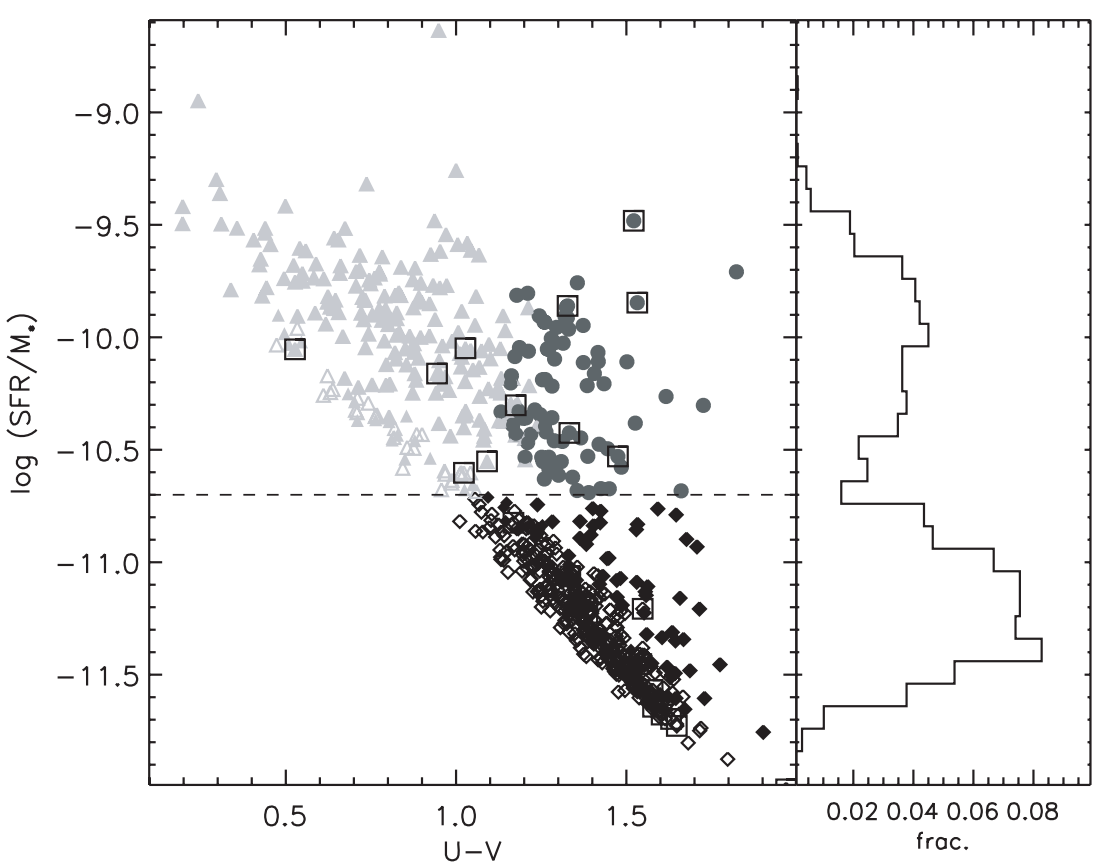

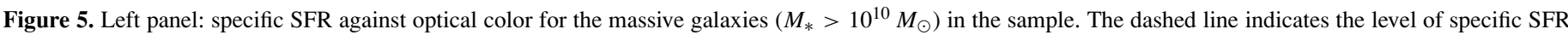

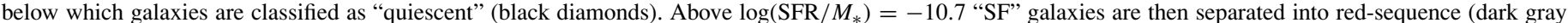

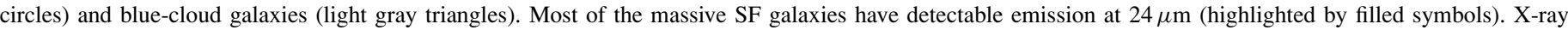

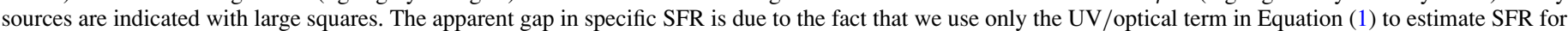

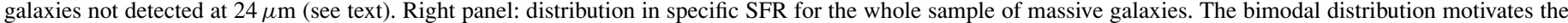
choice of a cut in specific SFR at $\log \left(\mathrm{SFR} / M_{*}\right)=-10.7$.

two peaks separate at a value of $\sim-10.7$, which is also very close to the mean value of specific SFR for this sample. This is clearly shown in the right panel of Figure 5. As discussed in Section 2.2, for galaxies with IR flux below the upper limit of $83 \mu \mathrm{Jy}$ we estimate SFR only from their UV luminosity. If we included the IR term also for these galaxies the distribution would no longer be bimodal, and the mean value of specific SFR would be $\log \left(\mathrm{SFR} / M_{*}\right) \sim-10.6$. We decide to keep the conservative approach of using the lower-limit SFR for galaxies not detected at $24 \mu \mathrm{m}$, however we will mention when relevant how the results would change if we used instead the upper-limit SFR (i.e., adopting the $24 \mu \mathrm{m}$ upper-limit flux of $83 \mu \mathrm{Jy}$ to estimate $L_{\mathrm{IR}}$ for non-detections).

Figure 5 (left panel) describes our classification for the 689 massive galaxies in the sample, showing their distribution in specific SFR against the rest-frame $U-V$ color. Quiescent galaxies (below $\log \left(\mathrm{SFR} / M_{*}\right)=-10.7$, dashed line) are shown as black diamonds and almost all of them belong to the red sequence. SF galaxies (above the dashed line) are distinguished into blue-cloud galaxies (light gray triangles), and red-sequence galaxies (dark gray circles). About $60 \%$ of the sample is classified as quiescent (406 galaxies), the remaining is divided into 77 red SF and 206 blue SF galaxies. Galaxies that have a detection at $24 \mu \mathrm{m}$ are highlighted with filled symbols. We note that all the red SF galaxies have $24 \mu \mathrm{m}$ detection, while $13 \%$ of the blue SF galaxies are not MIPS-detected (their UV-based SFR is thus more properly a lower limit to the total SFR). Among the quiescent galaxies, 81 have detectable IR emission. Few of the $24 \mu \mathrm{m}$ detected quiescent galaxies are assigned a specific SFR higher than expected on the basis of their color, but it is not clear whether the IR emission in these cases is truly indicative of low level of star formation or rather comes from circumstellar dust in red giant stars (but see Temi et al. 2007, 2008) or from an AGN (although none of these galaxies is associated with an
X-ray source, as shown by the large squares). In any case, even assuming that the IR emission in these galaxies is associated with young stars it is not enough to classify them as star forming.

It is worth mentioning that the location of galaxies in the specific SFR versus $U-V$ plane is independent of environment, with only the relative importance of blue-SF/red-SF/quiescent galaxies changing with environment, as we discuss in Figure 6.

The apparent gap in specific SFR in Figure 5 between IRdetected and IR-undetected galaxies is due to the drop of the $L_{\mathrm{IR}}$ term in Equation (1) in the latter case. Adopting an IR luminosity for IR-undetected galaxies given by the upper-limit flux of $83 \mu \mathrm{Jy}$ would increase the specific SFR of these galaxies and fill in the gap somewhat. While this would have a small effect on the number of blue SF galaxies (because their SFRs are already above the threshold even when not detected at $24 \mu \mathrm{m})$, the number of red-sequence galaxies classified as star forming would increase at the expense of quiescent galaxies. More quantitatively, adopting the upper limits on SFR and a specific SFR threshold of $\log \left(\mathrm{SFR} / M_{*}\right)=-10.7$ (as in our default case) or -10.6 (the mean value for the "upper limit" specific SFRs), the number of red SF galaxies would increase to 170 or 126 , respectively, while the number of quiescent galaxies would decrease to 299 or 347 , respectively (note that in this case the selection would be more sensitive to the exact cut in specific SFR adopted).

We certainly expect a number of SF galaxies to have colors as red as red-sequence galaxies simply due to inclination effects. We have visually inspected the STAGES and GEMS $V$-band images of the red SF galaxies in our sample. We found that $19 \%$ of them appear as edge-on spirals with dust lanes on the plane of the disk. These galaxies might be classified as blue SF if viewed with a different angle. Another $10 \%$ of the red SF galaxies are inclined spirals but with irregular structure (also in the dust), so it is not clear what the inclination effects in 

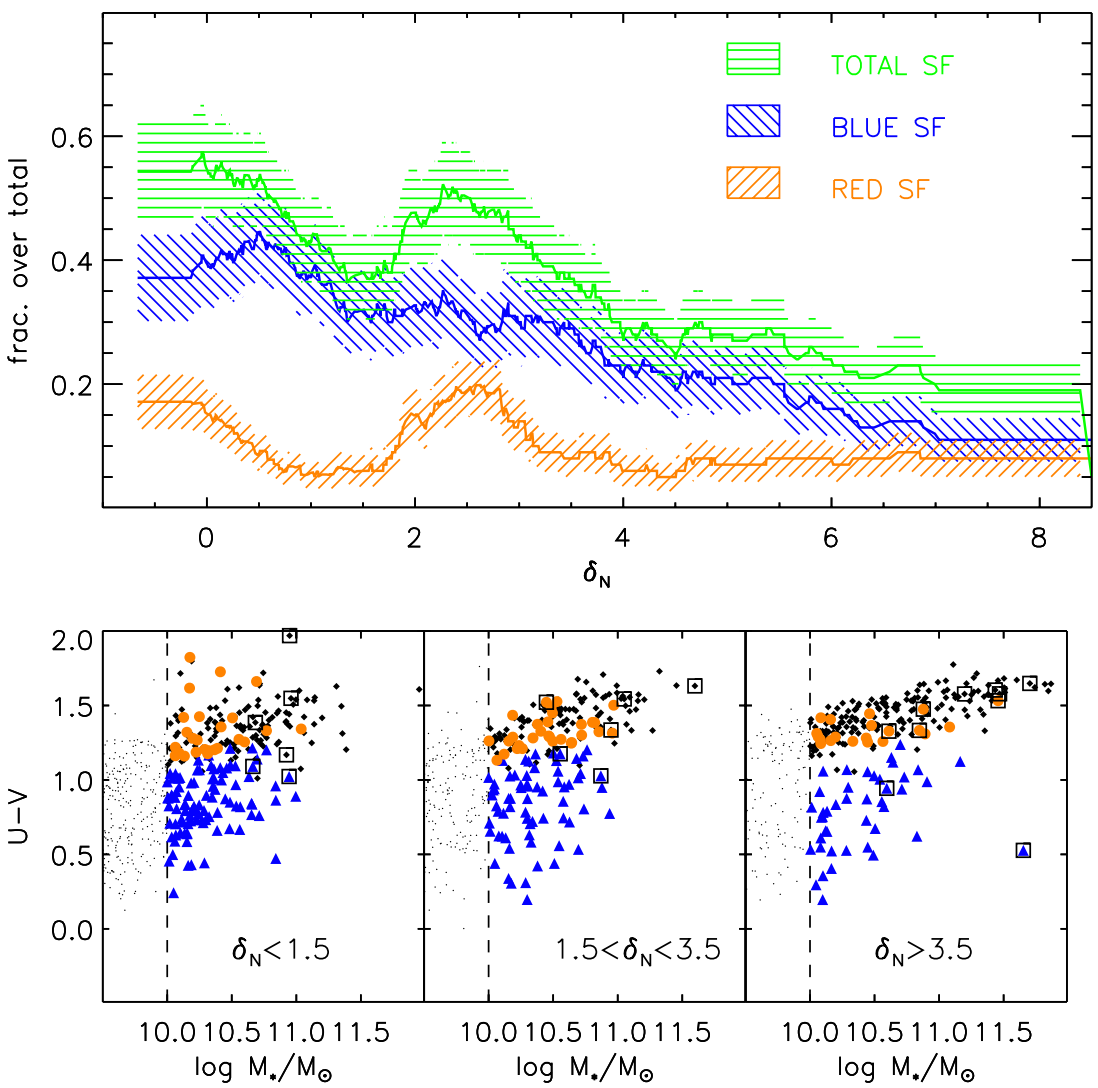

Figure 6. Bottom panels: rest-frame $U-V$ color against stellar mass for galaxies in low-density environments (left) compared with intermediate-density and highdensity environments (middle and right). Above $10^{10} M_{\odot}$ (dotted line) we distinguish quiescent galaxies (black diamonds), blue SF (blue triangles), and red SF galaxies (orange circles). Identified X-ray sources are marked with a square. Upper panel: fraction of unobscured (blue curve) and obscured (orange curve) SF galaxies above $10^{10} M_{\odot}$ as a function of galaxy number overdensity $\delta_{N}$. The green curve shows the total fraction of SF galaxies (i.e., the sum of the orange and the blue curves). In each case the shaded region encloses the $\pm 1 \sigma$ Poisson uncertainty. X-ray sources among SF galaxies have been excluded, but including them would make a negligible difference. Contrary to blue SF galaxies, whose fraction decreases monotonically with $\delta_{N}$, red SF galaxies are found preferentially at intermediate densities where they constitute $\sim 20 \%$ of the whole population, i.e., only slightly lower than blue SF galaxies.

(A color version of this figure is available in the online journal.)

these cases are. We conclude that undisturbed edge-on spirals can account for no more than $30 \%$ of the red SF galaxies in our sample. There must be an excess population that accounts for the full sample of red SF galaxies, either old galaxies with some residual star formation or galaxies with inclination-independent dust obscuration or a combination of both, as we discuss in Section 3.3.

\subsection{Galaxy Fractions Versus Environmental Density}

It is not obvious from Figure 4 whether the abundance of $24 \mu \mathrm{m}$ sources "hidden" among red-sequence galaxies is a feature characteristic of the cluster or whether these sources represent a ubiquitous population. We explore the possible environmental dependence in Figure 6. Here we do not separate galaxies between "cluster" and "field," instead we use the continuous definition of environment given by Equation (2). The lower panels of Figure 6 show the relation between optical color and stellar mass for galaxies in three disjoint density regimes, namely low-density environments with $\delta_{N}<1.5$, intermediate-density environments with $1.5<\delta_{N}<3.5$, and high-density environments with $\delta_{N}>3.5$. Different symbols distinguish the three classes of galaxies defined above (galaxies associated with an X-ray source are indicated with a square): quiescent galaxies (black diamonds), blue SF galaxies (blue triangles), and red SF galaxies (orange circles). We are particularly interested in the latter class of galaxies, which represents the class of obscured SF galaxies, in comparison to the "unobscured" class, i.e., those galaxies identified as star-forming also in the optical. Red SF galaxies tend to populate the low-mass end of the red sequence and their mass range does not evolve with environment, as opposed to quiescent galaxies. At fixed stellar mass, red SF galaxies are on average bluer than quiescent galaxies. We will explore these properties in Section 3.3.

The upper panel of Figure 6 shows the fraction of blue (unobscured) SF and of red (obscured) SF galaxies among all $M_{*}>10^{10} M_{\odot}$ galaxies as a function of density. Galaxy fractions are calculated as follows. We first order galaxies with increasing $\delta_{N}$ values. For each galaxy we then consider the neighboring galaxies within a given window in density $( \pm 0.5$ of the central value) and calculate the fraction of a given type of galaxies among this subsample. For galaxies in the first half bin of $\delta_{N}$ we do not measure fractions but we set their values to the first value actually measured (at $\delta_{N}=-0.5$ ). The width of the density bin is kept constant until a sufficient number (100) of galaxies fall in that bin. At higher densities, where the sampling is sparser, we let the bin width vary in order to enclose 100 neighboring galaxies (50 at lower densities and 50 at higher densities). ${ }^{20}$ When there are not anymore enough neighboring galaxies we set the fractions to the last measured values (this

\footnotetext{
${ }_{20}$ The density range remains constant up to $\delta_{N} \sim 4$, it increases to \pm 1 around $\delta_{N} \sim 5$. Above $\delta_{N}=5$ the density range probed is skewed toward higher densities, but the contamination by lower density galaxies does not increase.
} 


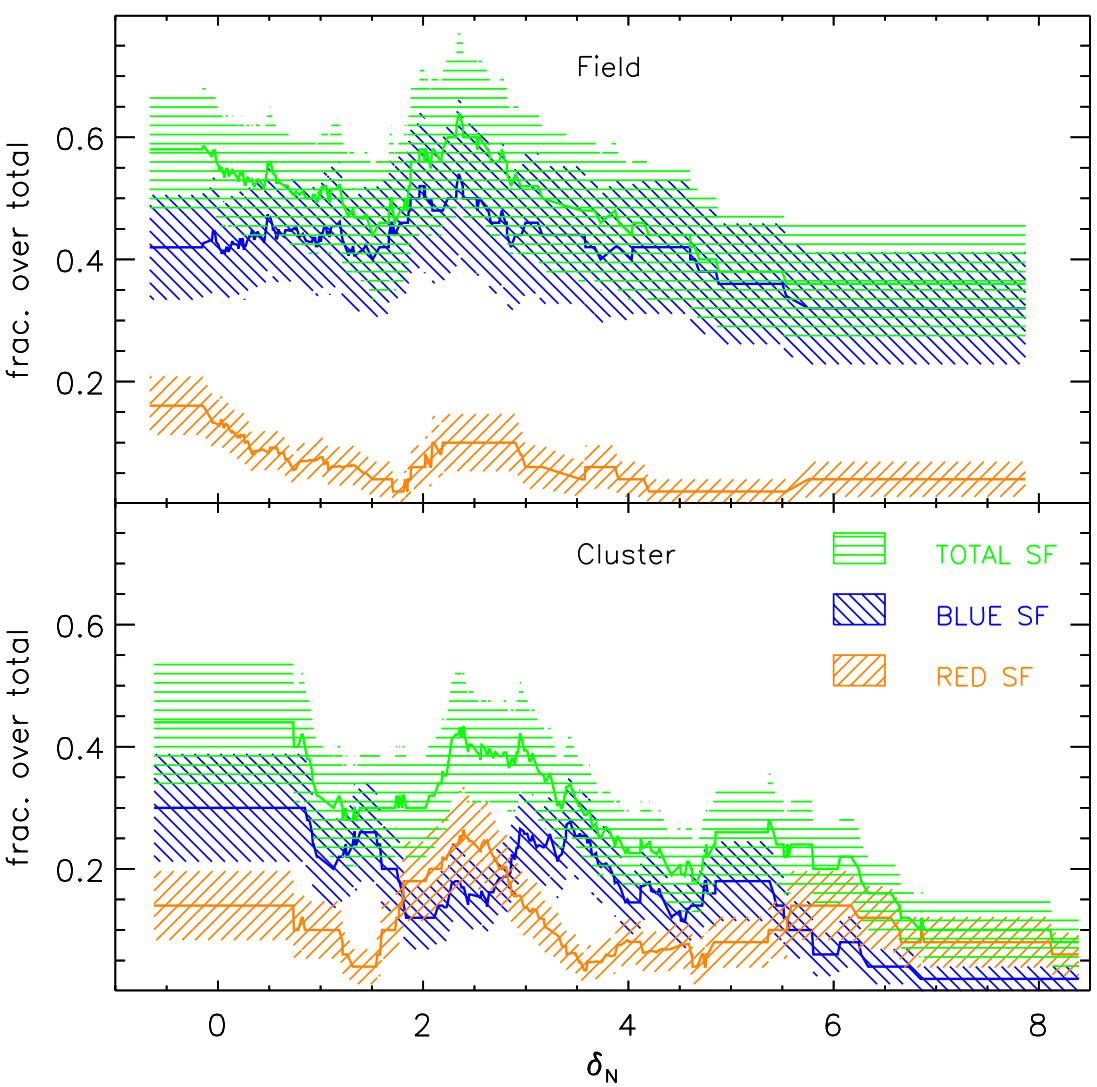

Figure 7. Fraction of obscured (orange), unobscured (blue), and all SF galaxies (green) as a function of density $\delta_{N}$ for the cluster (lower panel) and for the field sample (upper panel) separately. The relative abundance of red SF galaxies depends on both the local galaxy number density and the larger-scale environment: the excess of red SF galaxies at intermediate densities is much clearer in the cluster sample.

(A color version of this figure is available in the online journal.)

happens around a $\delta_{N}$ of 7). This procedure assures a signal-tonoise ratio of at least 10 with small variation along the density axis. The shaded regions in the upper panel of Figure 6 represent the Poisson uncertainty in the calculated fractions.

The blue curve in Figure 6 shows the environmental trend of the fraction of unobscured SF galaxies. As expected this fraction decreases significantly with density, from $\sim 40 \%$ at the low densities typical of the field to $\sim 10 \%$ in the densest environments of the cluster. This trend reflects the well known decrease in the number of SF galaxies in clusters. When we add the contribution of SF galaxies that are on the red sequence, the overall fraction of SF galaxies among massive galaxies (green curve) is increased over the entire density range covered. What is interesting is that the contribution added by red SF galaxies is not constant with $\delta_{N}$, but produces an enhancement in the SF fraction in particular at densities $1.5 \lesssim \delta_{N} \lesssim 4$.

The orange curve in Figure 6 shows the variation with density of the fraction of red SF galaxies. Contrary to blue SF galaxies, the decrease in the fraction of red SF galaxies with density is not monotonic. At the lowest densities of the field, red SF galaxies represent about $15 \%$ of the total. After an initial decrease from the field toward higher densities, the fraction of red SF galaxies increases again to values between $15 \%$ and $25 \%$ over the density range $2 \lesssim \delta_{N} \lesssim 3$, and then it settles to a value of $\lesssim 10 \%$ up to the highest densities of the cluster. The bottom line of Figure 6 is such that red SF galaxies represent a non-negligible fraction of the whole galaxy population even at intermediate and high densities. In particular, there is an overabundance of red SF galaxies at intermediate densities where their contribution is comparable to that of blue SF galaxies.
We have checked how the trend of red SF galaxies versus $\delta_{N}$ would change if we changed the definition of "SF" galaxies. If we included the IR term based on the $24 \mu \mathrm{m}$ upper-limit flux in the SFR estimate for MIPS-undetected galaxies, there would be an overall increase in the fraction of red SF galaxies. This would affect mainly the high-density environments (because of the higher abundance of red galaxies not detected at $24 \mu \mathrm{m}$, likely because genuinely old ellipticals), bringing the red SF fraction between $20 \%$ and $30 \%$ (the exact value depending on the specific SFR cut adopted). Even if this was correct, it would only strengthen our main point.

We also checked that the trend in the red SF fraction with density is robust against contamination by edge-on dusty spirals. Even by removing the $<30 \%$ contribution by galaxies identified as edge-on spirals (see Section 3.1), we still detect an overabundance of red SF galaxies at intermediate densities and the qualitative behavior with $\delta_{N}$ does not change.

In Figure 7 we show again the fraction of obscured and unobscured SF galaxies as a function of the continuous density measure $\delta_{N}$ as in Figure 6 but distinguishing galaxies belonging to the A901/902 cluster (lower panel) and those living in the field (upper panel). In the field sample alone there is only a weak signal of an excess of red SF galaxies at intermediate densities. The excess found in Figure 6 for the sample as a whole is largely driven by cluster galaxies. Figure 7 shows that red SF galaxies are a phenomenon more typical of the cluster environment, where their fraction is comparable to that of blue SF galaxies. Thus, not only the local galaxy number density but also the larger-scale environment plays a role in shaping the star formation activity and dust attenuation of galaxies. 


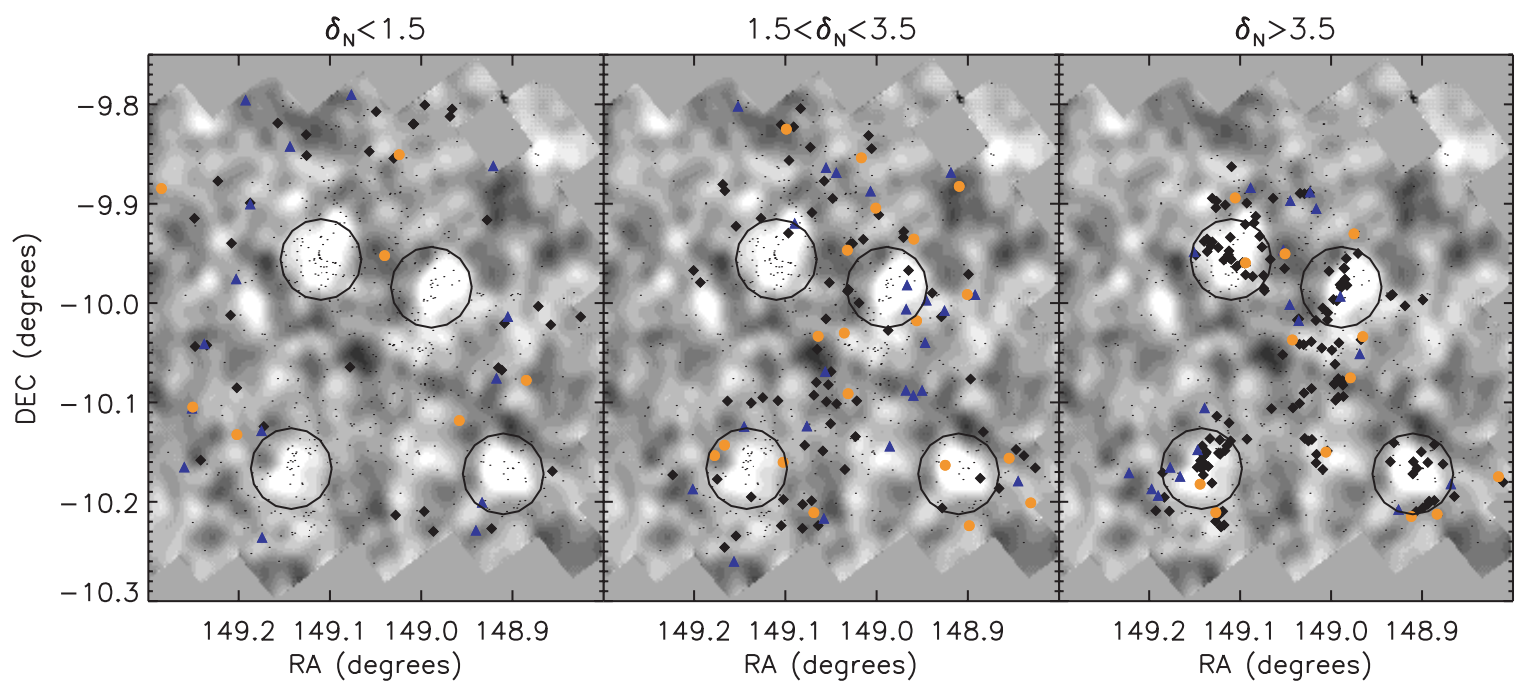

Figure 8. Position of quiescent (black diamonds), red SF (orange circles), and blue $\mathrm{SF}$ (blue triangles) galaxies in the A901/902 supercluster at overdensities $\delta_{N}<1.5$, $1.5<\delta_{N}<3.5$, and $\delta_{N}>3.5$. The underlying image reproduces the dark matter reconstruction of the supercluster as derived by Heymans et al. (2008), with intensity scaling as the surface mass density $\kappa$. The big circles indicate the four main supercluster structures (in clockwise order from top left A901a, A901b, SW group, A902).

Figure 8 illustrates the position on the sky of the cluster red SF galaxies (compared to blue SF and quiescent galaxies) in the three density ranges of Figure 6. The gray scale shows the dark matter map, as expressed by the surface mass density $\kappa$, reconstructed by Heymans et al. (2008) with the STAGES $H S T$ data. Low $\delta_{N}$ values are typical of the outskirts of the cluster, mainly populated by blue SF galaxies (left panel). High $\delta_{N}$ values are instead typical of the four main supercluster cores and of the filamentary structures connecting them, traced by the quiescent galaxy population (right panel). Red SF galaxies populate the medium-density regime, the infalling regions around the cluster cores, where episodes of obscured star formation might be favored (middle panel). This supports the analysis of Wolf et al. (2005), who identified an overabundance in the medium-density regions of the A901/902 supercluster of dusty, intermediate-age, red galaxies, classified on the basis of their location in optical color-color diagrams.

It is also of interest to ask what is the contribution in stellar mass and star formation activity of the different classes of galaxies. Figure 9 shows the fraction of stellar mass contributed by $M_{*}>10^{10} M_{\odot} \mathrm{SF}$ galaxies (green curve) as a function of environmental density. As in Figure 6 we distinguish SF galaxies on the red sequence (orange curve) and on the blue cloud (blue curve). The stellar mass fraction is calculated in the same way as the number fractions shown in Figure 6, but weighting each galaxy by its stellar mass. The decline from low to high densities of the stellar mass fraction contributed by SF galaxies reflects the decline in their number density. The blue and orange dotted lines reproduce the number fraction of blue SF and red SF galaxies, respectively. At all $\delta_{N}$ the fraction in mass of SF galaxies, either obscured or unobscured, is lower than the corresponding fraction in number. This comes from the fact that SF galaxies are preferentially less massive than quiescent, elliptical galaxies. The effect becomes stronger at high densities (at least for red SF galaxies), where the mass function of quiescent early-type red-sequence galaxies extends to higher masses. At low and intermediate densities the difference between the number and stellar mass fractions is lower for red SF galaxies than for blue SF galaxies, indicating a different stellar mass distribution of the two classes of galaxies, as we will show in Section 3.3.

In Figure 10, we investigate the amount of obscured star formation over the total star formation activity as a function

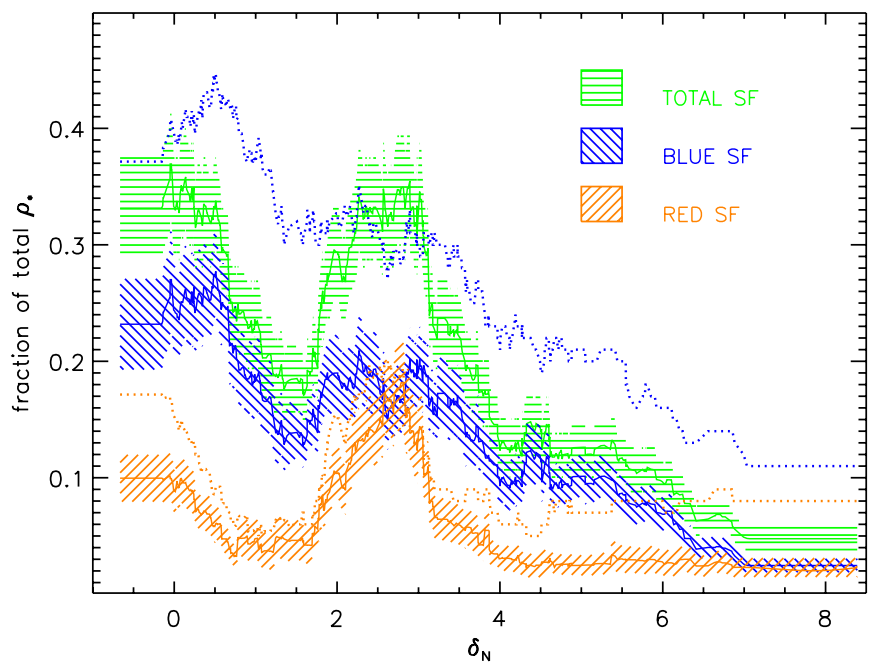

Figure 9. Fractional contribution to the total stellar mass density from the sample of massive SF galaxies as a whole (green), and split into blue SF (blue) and red SF (orange). The hatched area around each curve indicates the associated uncertainty, calculated assuming a 0.3 dex error in stellar mass. For comparison, the blue and orange dotted lines reproduce the number fractions of blue SF and red SF galaxies, respectively, as shown in Figure 6.

of environment. This is calculated as the fraction, weighted by SFR, of red SF galaxies over all SF galaxies, and is shown by the solid curve and hatched region. For comparison, the dotted curve shows the number fraction of red SF galaxies among all SF galaxies. As expected the majority of the starformation activity resides in galaxies populating the blue cloud, independently of environment. Nevertheless, there is a nonnegligible contribution, both in number and in total SFR, from obscured SF galaxies. In particular, there is a clear excess of obscured star formation at intermediate densities $\left(2 \lesssim \delta_{N} \lesssim 4\right)$, where red SF galaxies constitute up to $40 \%$ of all $\mathrm{SF}$ galaxies and contribute between $25 \%$ and $35 \%$ of the whole star formation activity at those densities. At higher densities, red SF galaxies still make up 40\% of the whole SF class at these densities, but their contribution to the total star formation activity goes down to $\sim 20 \%$. This suggests a small but detectable suppression of the SFR of high- 


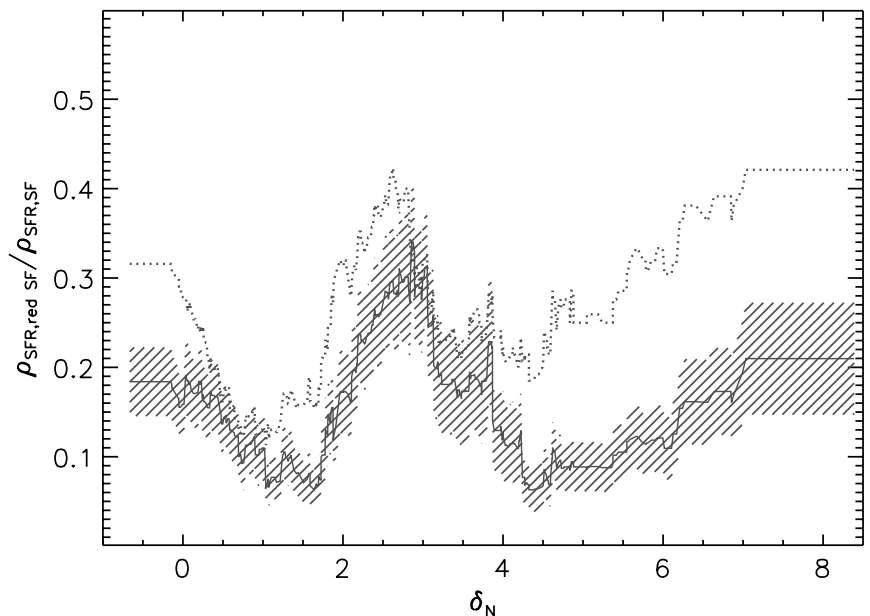

Figure 10. Fraction of SFR density contributed by red-sequence SF galaxies. This is compared to their number fraction over the whole SF population (dotted line). The hatched region represent the uncertainty, calculated assuming a 0.3 dex error on the SFR estimates.

density red SF galaxies compared to their intermediate-density counterparts.

Finally, Figure 11 illustrates the amount of contamination on the red sequence from obscured SF galaxies. This is expressed both in terms of the stellar mass fraction (solid line and hatched region) and of the number fraction (dotted line) of SF galaxies among red-sequence galaxies. At low densities, SF galaxies contribute roughly $15 \%$ in stellar mass and $30 \%$ in number to the red sequence. This fraction is in agreement with studies of the mix in the morphology and star formation activity of the "field" red sequence at different redshifts (e.g., Franzetti et al. 2007; Cassata et al. 2007, 2008). As expected from the general decrease in the number of SF galaxies in dense environment, the contamination of the red sequence by SF galaxies also decreases with density. However, it reaches values of a few percent only at the highest densities of the cluster, where the red sequence is highly dominated by quiescent galaxies. At intermediate densities, instead, there is an excess of (preferentially obscured) star formation, as already discussed in the previous figures.

\subsection{The Properties of Red SF Galaxies}

In this section we compare the star formation and morphological properties of red-sequence SF galaxies to those of blue-cloud SF and quiescent galaxies. We also investigate any environmental variation of such properties in SF galaxies. A follow-up analysis by Wolf et al. (2008) based on STAGES data presents environmental trends of the properties of cluster galaxies by distinguishing (visually classified) morphological types and SED types.

Figure 12 shows the distributions in stellar mass, specific SFR, and total SFR of red SF galaxies (hatched histograms) in three density regimes $\left(\delta_{N}<1.5,1.5<\delta_{\mathrm{N}}<3.5, \delta_{N}>3.5\right)$. In each density range, these distributions are compared to those of blue SF galaxies (gray shaded histograms) and quiescent galaxies (dashed histograms). The left panels of Figure 12 show that, while the stellar mass of quiescent galaxies clearly increases from low to high densities, the stellar masses of SF galaxies hardly vary with density, almost independently of their obscuration level. There is however a hint that the mean stellar mass of red SF galaxies at intermediate densities is slightly higher than that of their low-density and highdensity counterparts $\left(\left\langle\log \left(M_{*} / M_{\odot}\right)\right\rangle=10.47 \pm 0.05\right.$ com-

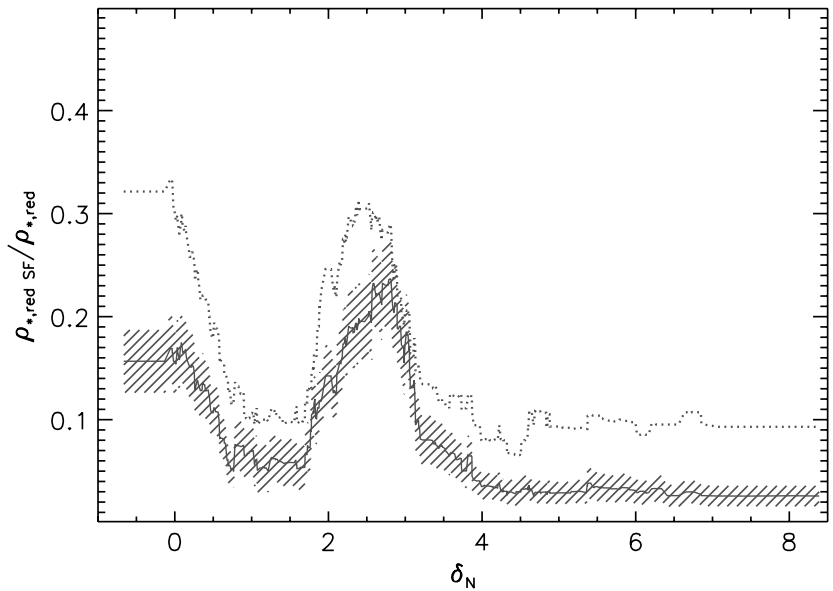

Figure 11. Fraction of the stellar mass on the red-sequence contributed by obscured SF galaxies with $M_{*}>10^{10} M_{\odot}$ (solid curve and hatched region). This is compared to their number fraction over all massive red-sequence galaxies.

pared to $\left\langle\log \left(M_{*} / M_{\odot}\right)\right\rangle=10.32 \pm 0.05$ and $\left\langle\log \left(M_{*} / M_{\odot}\right)\right\rangle=$ $10.35 \pm 0.08$ at low and high densities, respectively). The distribution in stellar mass at intermediate $\delta_{N}$ compares with that at low $\delta_{N}$ with a Kolmogorov-Smirnov $(\mathrm{K}-\mathrm{S})$ test probability of 0.03 that the two distributions are drawn from the same parent distribution. The $\mathrm{K}-\mathrm{S}$ test between the distribution at intermediate $\delta_{N}$ and at high $\delta_{N}$ gives a probability of 0.2 . Also, red SF galaxies at intermediate densities are on average more massive than blue SF galaxies at the same densities (which have $\left.\left\langle\log \left(M_{*} / M_{\odot}\right)\right\rangle=10.34 \pm 0.03\right)$, with a $\mathrm{K}-\mathrm{S}$ probability of 0.05 .

The second and third columns of plots in Figure 12 show the distributions in specific SFR and total SFR, respectively. For completeness we also show here the measured SFR of quiescent galaxies, which have by definition specific SFR lower than $2 \times 10^{-11} \mathrm{yr}^{-1}$. We do not detect any significant variation with environment in the SFR of blue-cloud SF galaxies. Also the specific SFR of red SF galaxies appears independent of environment, but their average SFR at intermediate densities is slightly higher than at low and high densities $(\langle\log \mathrm{SFR}\rangle=0.22 \pm 0.07$ compared to $\langle\log \mathrm{SFR}\rangle=$ $0.07 \pm 0.06$ and $\langle\log \mathrm{SFR}\rangle=0.01 \pm 0.08$, respectively), as a consequence of the slightly higher $M_{*}$ discussed above.

As a general remark, it is interesting to note that the red, often IR-bright, SF galaxies in our sample are not experiencing a burst of star formation. They have instead less intense star formation activity compared to blue-cloud galaxies, independently of environment. Their average specific SFR is from 0.2 dex to 0.3 dex lower than blue SF galaxies at the $5 \sigma$ level (the $\mathrm{K}-\mathrm{S}$ test on their specific SFR distributions provides a probability of $0.01,0.001$, and 0.006 at low, intermediate, and high densities, respectively).

The right panels of Figure 13 show the distribution in the $V$ band Sérsic index $n$ for the three classes of galaxies in the three density regimes. As expected, the distribution of SF galaxies peaks at low values of $n$ indicating that in all environments star formation occurs preferentially in disk-dominated systems. This result holds for both unobscured and obscured SF galaxies, which have similar distributions in the Sersic index. Also in this case we might witness a difference only at intermediate densities (although with rather low significance), where red SF galaxies tend to be more bulge-dominated than blue SF galaxies (with a $\langle n\rangle=2.28 \pm 0.35$ compared to $\langle n\rangle=1.76 \pm 0.21$, and a K-S probability of 0.12 for the two distributions to be the same). 


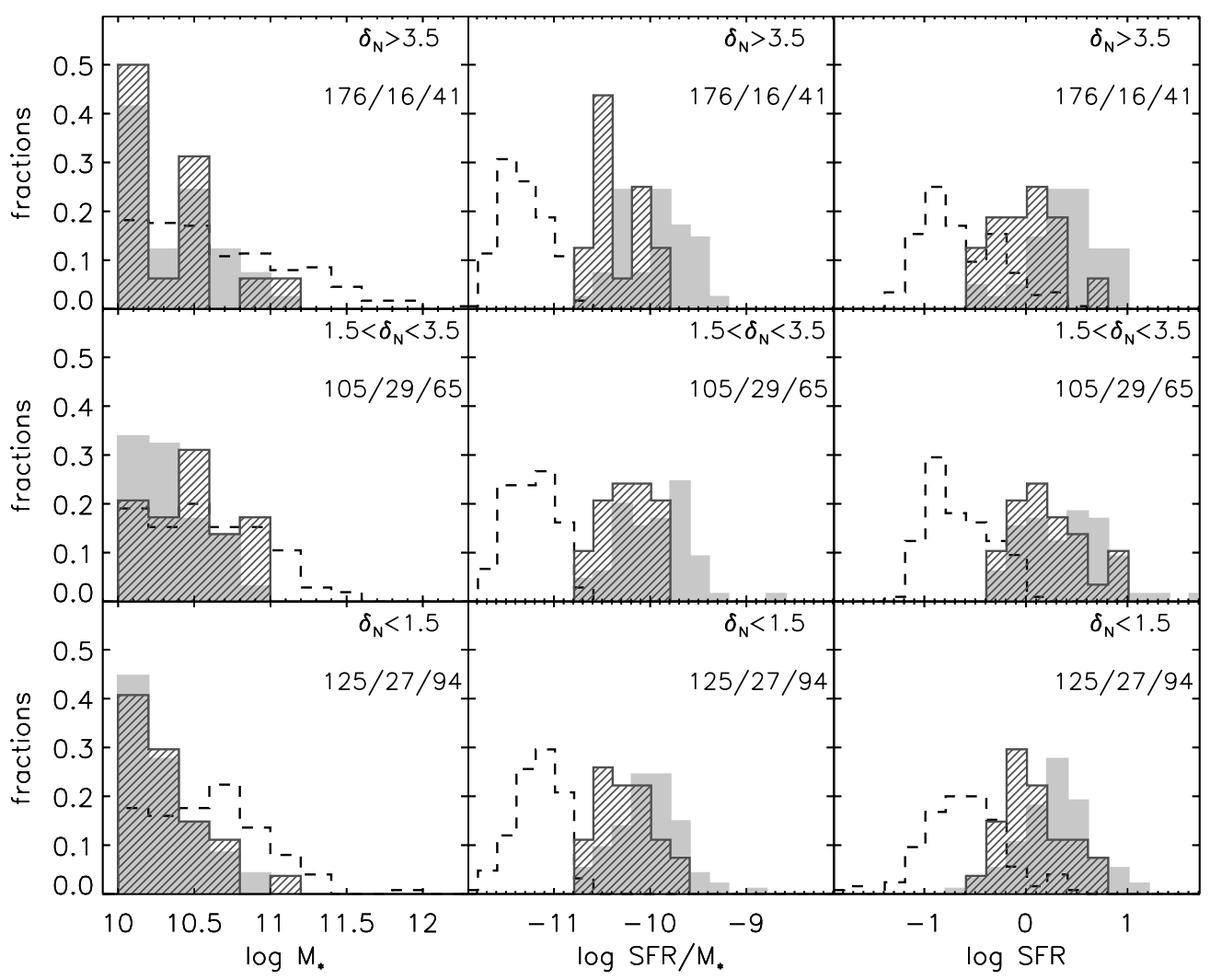

Figure 12. From left to right: distribution in stellar mass, SFR per unit mass, and total SFR for different classes of galaxies in three density regimes (increasing from bottom to top as indicated in each panel). Galaxies are divided into quiescent (black dashed histograms), blue star-forming (gray shaded histograms), and red SF galaxies (dark gray hatched histograms). As in Figure 6, X-ray sources identified among SF galaxies are excluded. The total number of quiescent/red-SF/blue-SF galaxies in each density range is also indicated in each panel. The histograms in each panel are normalized by the number of galaxies in the corresponding class and in the corresponding density bin.

In this work, we have defined SF galaxies as "obscured" or "unobscured" only on the basis of their optical color, namely whether they fall redward or blueward of the red-sequence cut, respectively. The dust attenuation of the UV flux in SF galaxies is often quantified by the ratio of the IR to UV luminosities (e.g., Gordon et al. 2000). ${ }^{21} \mathrm{We}$ then look at the dust attenuation properties of red $\mathrm{SF}$ and blue $\mathrm{SF}$ galaxies, as expressed by the IR-to-UV luminosity ratio, $\log \left(L_{\mathrm{IR}} / L_{\mathrm{UV}}\right)$. This is shown in the left panels of Figure 13 for red and blue SF galaxies in the same three density regimes as Figure 12 (for completeness we include also quiescent galaxies; dashed histograms). The distributions are shown only for galaxies with a detection at $24 \mu \mathrm{m}$ (for each $\delta_{N}$ bin the total number of galaxies in each class is indicated in the panels). All red SF galaxies are detected at $24 \mu \mathrm{m}$, while $13 \%$ of the massive blue-cloud galaxies have $24 \mu \mathrm{m}$ flux below the detection limit. The fraction of blue-cloud galaxies missed is however independent of mass. The $24 \mu \mathrm{m}$ selection in this plot affects only quiescent galaxies, whose detection rate decreases with mass.

As expected, in any density range, red-sequence SF galaxies have on average higher $L_{\mathrm{IR}} / L_{\mathrm{UV}}$ than blue-cloud galaxies, indicating a higher level of dust attenuation. The distri-

\footnotetext{
21 The conversion from $L_{\mathrm{IR}} / L_{\mathrm{UV}}$ to $\mathrm{UV}$ attenuation depends on the galaxy star formation activity and the stellar age (Cortese et al. 2008), but this should not be a concern for the following discussion. First, the IR luminosity that we infer is based on the luminosity at $24 \mu \mathrm{m}$, hence relatively insensitive to the (typically colder) dust heated by old stars. Second, the red SF galaxies in our sample span only 1 order of magnitude in specific SFR, hence the $L_{\mathrm{IR}} / L_{\mathrm{UV}}$ can at least give us an insight into the relative dust attenuation among these galaxies.
}

bution in $\log \left(L_{\mathrm{IR}} / L_{\mathrm{UV}}\right)$ differ most significantly at low and intermediate densities (with a K-S probability of 0.003 and 0.002 , respectively). In the lowest-density bin, red SF galaxies have an average $\log \left(L_{\mathrm{IR}} / L_{\mathrm{UV}}\right)$ of $1.07 \pm 0.09$, compared to the $0.68 \pm 0.04$ of blue SF galaxies. At intermediate densities the average $\log \left(L_{\mathrm{IR}} / L_{\mathrm{UV}}\right)$ of red SF galaxies is $1.04 \pm 0.07$ compared to $0.73 \pm 0.05$ of blue SF galaxies. At the highest densities of the cluster, red SF galaxies still have higher dust attenuation with respect to blue SF galaxies (with a $\left\langle\log \left(L_{\mathrm{IR}} / L_{\mathrm{UV}}\right)\right\rangle=0.87 \pm 0.08$ compared to $0.6 \pm 0.05$ of blue SF), although the difference between the two distributions is less significant (with a $\mathrm{K}-\mathrm{S}$ probability of 0.12 ).

Contrary to the other parameters analyzed so far, the IR-toUV luminosity ratios of red SF galaxies appear to have a roughly bimodal distribution, with a peak around $\log \left(L_{\mathrm{IR}} / L_{\mathrm{UV}}\right)$ values similar to the main population of blue SF galaxies and another peak at significantly higher values. This is particularly evident at low densities but seems to persist in all environments with varying proportion between the two groups of red SF galaxies. We can explicitly distinguish red SF galaxies on the basis of their IR-to-UV luminosity ratio, choosing a cut at $\log \left(L_{\mathrm{IR}} / L_{\mathrm{UV}}\right)=1$. By doing so, we find out that low-attenuation red SF galaxies differ from high-attenuation red SF galaxies in their specific SFR, their morphology and their environmental dependence, suggesting that different evolutionary mechanisms are acting on them.

Low-attenuation red SF galaxies have systematically lower specific SFR than high-attenuation red SF galaxies (with an average $\log \left(\mathrm{SFR} / M_{*}\right)$, over all environments, of $-10.43 \pm 0.03$ 


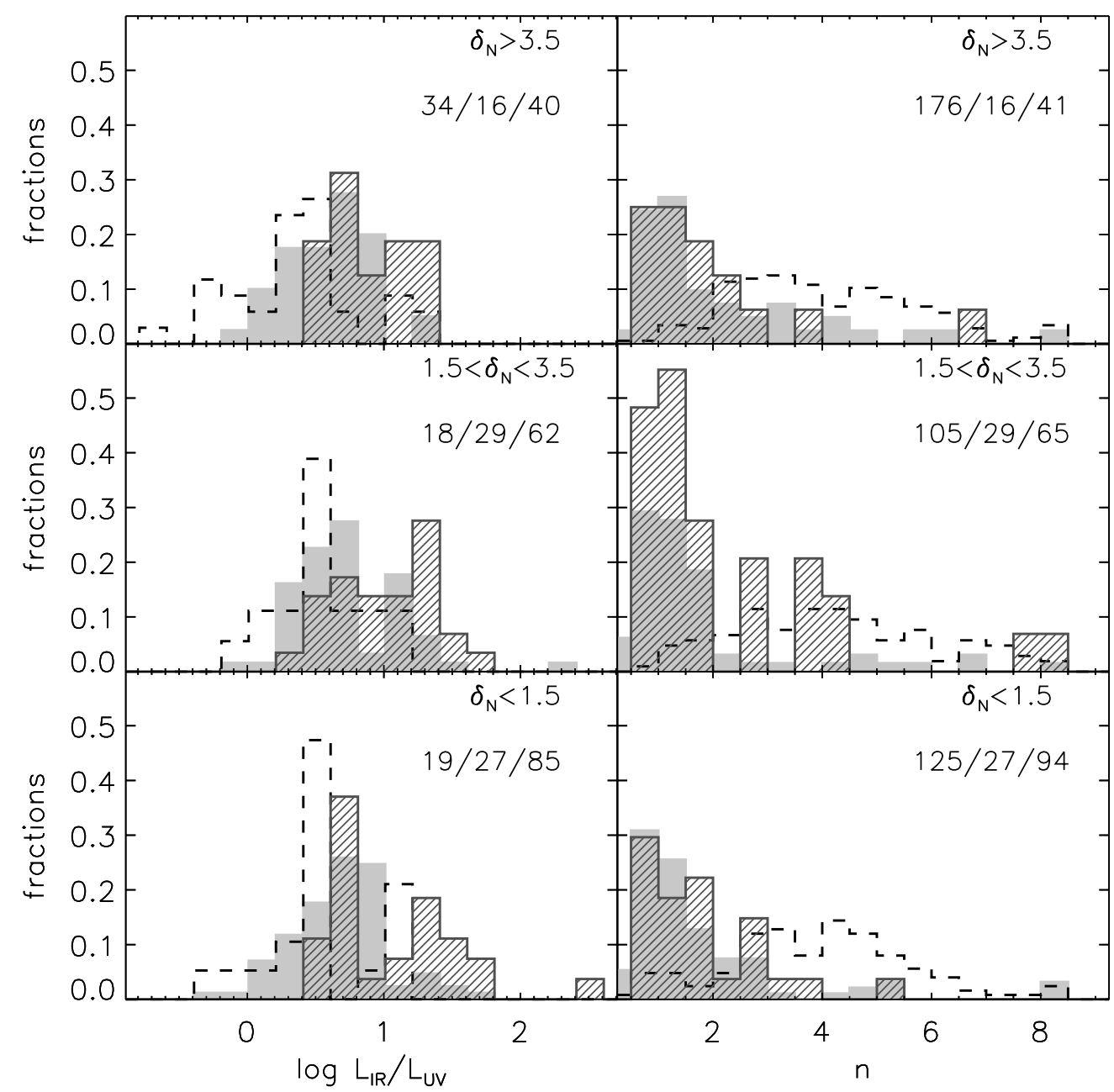

Figure 13. Distribution in the IR-to-UV luminosity ratio (left) and the $V$-band Sersic index $n$ (right) for quiescent (black dashed histograms), blue SF (gray shaded histograms), and red SF (dark gray hatched histograms) galaxies in the same density regimes as in Figure 12 . Note that the distributions in $L_{\mathrm{IR}} / L_{\mathrm{UV}}$ are calculated only for galaxies with a detection at $24 \mu \mathrm{m}$, not upper limits. This affects only the distribution of quiescent galaxies, that have a low IR detection rate which decreases with mass. The total number of quiescent/red-SF/blue-SF galaxies in each density range is also indicated in each panel. The histograms in each panel are normalized by the number of galaxies in the corresponding class and in the corresponding density bin.

compared to $-10.04 \pm 0.04$ for the latter class). The distributions in specific SFR of low-attenuation and high-attenuation red SF galaxies differ most significantly at low and intermediate densities (with a $\mathrm{K}-\mathrm{S}$ test probability of 0.004 and 0.002 , respectively). Moreover, although with less significance, it is interesting to note that low-attenuation red SF galaxies tend to be fitted by higher values of the Sérsic index than highattenuation red SF galaxies (with a $\langle n\rangle=2.43 \pm 0.96$ compared to $\langle n\rangle=1.54 \pm 0.42$ for the latter class, averaged over all densities). The morphology of both galaxy classes, at least as quantified by $n$, is not a function of environment.

The fraction of low-attenuation red SF galaxies over the entire population of SF galaxies varies from $11.6 \pm 3 \%$ at low densities to $14.9 \pm 4 \%$ at intermediate densities and $17.5 \pm 6 \%$ at high densities. There might be a tendency of low-attenuation red SF galaxies becoming progressively more frequent in high-density environments, but the errors make these fractions consistent with being independent of environment. In contrast, highattenuation red SF galaxies appear to be more abundant at intermediate densities at about the $2 \sigma$ level: at intermediate densities they represent $16 \pm 4 \%$ of all SF galaxies, compared to $10.7 \pm 3 \%$ at low densities and $10.5 \pm 4 \%$ at high densities. Their stellar mass is also slightly higher at intermediate densities $\left(\left\langle\log \left(M_{*} / M_{\odot}\right)\right\rangle=10.52 \pm 0.07\right.$ compared to $10.33 \pm 0.06$ and $10.28 \pm 0.1$ at low and high densities, respectively).

It is worth noting that also among blue SF galaxies there is a subsample of galaxies with $\log \left(L_{\mathrm{IR}} / L_{\mathrm{UV}}\right)>1$. By selecting high-attenuation SF galaxies independently of optical color, the same picture emerges in comparison to low-attenuation red $\mathrm{SF}$ galaxies as outlined above. Indeed, the argument of an overabundance at intermediate densities of dust-obscured star formation would be even stronger: the fraction of $\log \left(L_{\mathrm{IR}} / L_{\mathrm{UV}}\right)>1$ SF galaxies among all SF galaxies would be $34 \pm 7 \%$ at intermediate $\delta_{N}$, compared to $18 \pm 4 \%$ at low $\delta_{N}$ and $16 \pm 6 \%$ at $\operatorname{high} \delta_{N}$.

This is further illustrated in Figure 14 which shows the fraction versus the galaxy overdensity $\delta_{N}$ of red SF galaxies, separated into low attenuation (upper panel) and high attenuation (lower panel). Low-attenuation red SF galaxies constitute on average $\sim 5 \%$ of the whole sample with no significant dependence on environment. The excess at intermediate densities of red-sequence star formation identified in Figure 6 is mainly contributed by high-attenuation red SF galaxies, which represent about $12 \%$ of the whole population at $\delta_{N} \sim 2.5$. Even excluding the edge-on spirals among high-attenuation red SF galaxies (see Section 3.1), the trend with $\delta_{N}$ is still consistent 


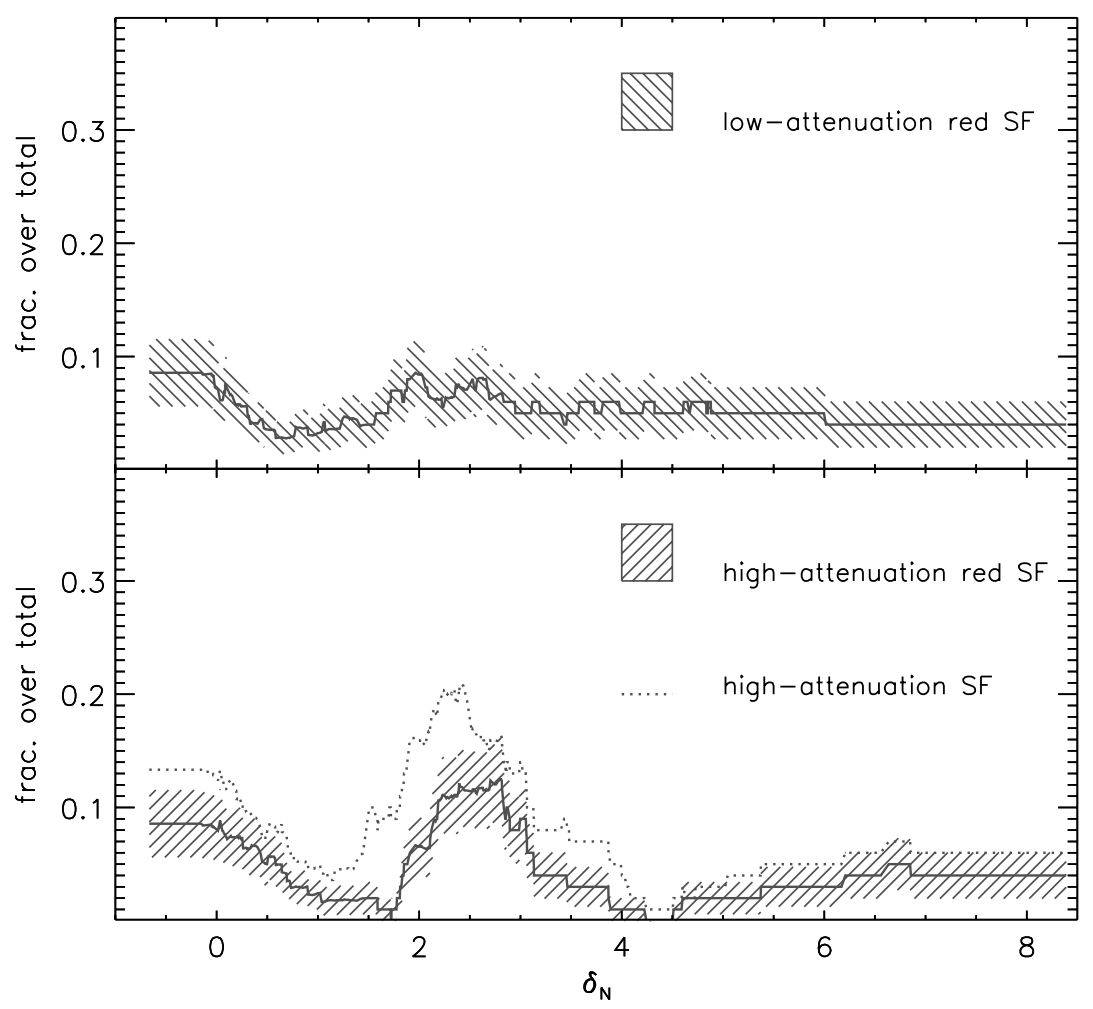

Figure 14. Upper panel: fraction of low-attenuation $\left(\log \left(L_{\mathrm{IR}} / L_{\mathrm{UV}}\right)<1\right)$ red SF galaxies over all galaxies as a function of overdensity $\delta_{N}$ : they represent on average $\sim 5 \%$ of the whole population, almost independent of environment. Lower panel: fraction of high-attenuation $\left(\log \left(L_{\mathrm{IR}} / L_{\mathrm{UV}}\right)>1\right)$ red SF galaxies vs. $\delta_{N}$ : there is an excess of dust-obscured star formation at intermediate densities. The same picture emerges if we consider all (blue and red) high-attenuation SF galaxies (dotted curve).

within the errors with that shown in Figure 14. Moreover, dustobscured star formation could represent up to $20 \%$ of the whole population at these densities by considering all SF galaxies with $\log \left(L_{\mathrm{IR}} / L_{\mathrm{UV}}\right)>1$, regardless of their optical color (dotted curve).

Based on the considerations above, we can say that lowattenuation red SF galaxies are likely spirals that are gradually quenching their star formation and appear bulge-dominated because of disk fading. They might resemble the anemic spirals found in local clusters as Coma and Virgo (van den Bergh 1976; Kennicutt 1983; Gavazzi et al. 2002, 2006). Some mechanism that removes gas on relatively long timescales could be responsible for their transformation toward quiescence. However, given their negligible environmental dependence, internal processes leading to star formation quenching are equally possible and maybe even sufficient. On the other hand, high-attenuation red SF galaxies are disk-dominated spirals affected by some mechanism, particularly efficient at intermediate densities, that triggers obscured episodes of star formation without significantly changing the morphology, at least on timescales over which star formation is still detectable.

\section{DISCUSSION AND CONCLUSIONS}

\subsection{Star Formation Among Red Galaxies}

We have combined сомво-17 optical data with MIPS $24 \mu \mathrm{m}$ data for a sample of low-redshift $(0.05<z<0.3)$ galaxies in the CDFS and A901 fields with the aim of studying the occurrence of obscured star formation as a function of environment. The $24 \mu \mathrm{m}$ information allows us to recover directly the flux from young stellar populations absorbed and re-emitted by dust, and thus to trace, in combination with the UV/optical information, the total (unobscured and obscured) star formation activity in galaxies. The A901 field is particularly suited from this kind of analysis, not only for the exceptional multiwavelength coverage, but also because it includes the complex A901/902 supercluster at $z=0.165$ extending over an area of $5 \times 5 \mathrm{Mpc}^{2} \mathrm{~h}_{70}^{-2}$. The supercluster is composed of four main substructures, probably in the process of merging. The complex dynamical state of the A901/902 supercluster potentially makes it an ideal case for identifying galaxies in their process of evolution under the influence of environment. The CDFS, with the same multiwavelength coverage, offers instead a control sample of field galaxies at similar redshift as the cluster.

In this work we have focused on galaxies with stellar masses larger than $10^{10} M_{\odot}$, above which the red sequence is complete out to $z=0.3$ (our limiting redshift). This mass limit roughly corresponds to $0.1 \times M^{*}$ over the redshift range $z<0.3$ (Bell et al. 2003; Borch et al. 2006). We define as star forming those galaxies with a specific SFR (derived from UV and IR luminosities) above $2 \times 10^{-11} \mathrm{yr}^{-1}$. Our focus is on SF galaxies populating the red sequence, either because they show low levels of star formation insufficient to alter the color of the underlying older population or because their star formation activity is highly obscured by dust. Studies based on the UV and optical emission of galaxies have identified a significant amount of low-level star formation in low-mass ellipticals (Yi et al. 2005; Kaviraj et al. 2007), with a hint of a peak in "frosting" activity at group densities (Rogers et al. 2007). Starformation indicators that are less sensitive to dust attenuation, such as the $24 \mu \mathrm{m}$ emission that we exploit in this work, are instead required to detect dust-obscured star formation.

We have studied the abundance of blue and red SF galaxies as a function of environment, as expressed by the galaxy number 
overdensity in a radius of $0.25 \mathrm{Mpc}$, focusing on the contribution of star formation on the red sequence compared to optically detectable star formation. Our results can be summarized as follows.

1. The overall fraction of SF galaxies decreases from $\sim 60 \%$ in underdense regions to $\sim 20 \%$ in high-density regions. The stellar mass fraction contributed by SF galaxies also decreases going from the field to the cluster cores. The decline is steeper than for the number fraction because, while no significant environmental evolution in stellar mass occurs for SF galaxies, the mass function of quiescent galaxies reaches higher stellar masses at higher densities.

2. The fraction of blue SF galaxies decreases monotonically from $\sim 40 \%$ at low densities to less than $20 \%$ at higher densities. In contrast, red $\mathrm{SF}$ galaxies do not show a monotonic behavior as a function of environment. After an initial decline of the red SF fraction from the field to higher $\delta_{N}$, we identify an overabundance of obscured star formation at intermediate densities, those typical of the outskirts of the A901/902 supercluster cores. At both intermediate and high densities, red SF galaxies represent $40 \%$ of all SF galaxies and contribute $20 \%-30 \%$ of the total star formation activity at these densities.

To a first order, our results confirm the well-known SFRdensity relation (e.g., Gavazzi et al. 2002; Lewis et al. 2002; Gómez et al. 2003; Balogh et al. 2004a; Kauffmann et al. 2004) and morphology-density relation (e.g., Dressler 1980; Dressler et al. 1997; Treu et al. 2003; van der Wel 2008). In addition to this, we find a significant contribution by red-sequence galaxies, identified as star forming through their IR emission, to the total star formation activity up to the highest densities of the cluster. This would be at least partly missed by optical studies. This result is consistent with Wolf et al. (2005) who already found an enhancement of optically classified dusty red galaxies in the medium-density outskirts of the A901/902 supercluster. In this work, supported by deep $24 \mu \mathrm{m}$ data, we directly measure the amount of star formation going on in these galaxies.

Our results are also in line with recent studies of clusters at similar redshifts as A901/902 or higher, which have identified a population of IR-bright galaxies in filaments and the infalling regions of the clusters. Fadda et al. (2000) found a population of $15 \mu \mathrm{m}$ detected galaxies with a high $15 \mu \mathrm{m}$-to-optical flux ratio suggesting star formation activity in the cluster A1689 at $z=0.18$, in excess with respect to the Virgo and Coma cluster (see also Duc et al. 2002). In the cluster A2667 at $z=0.23$, Cortese et al. (2007) have identified a IR-bright $L^{*}$ spiral galaxy in the process of being transformed by the cluster environment which triggers an intense burst of star formation. At similar redshift, Fadda et al. (2008) find two filamentary structures in the outskirts of the A1763 cluster at $z=0.23$ (probably undergoing accretion events), which are rich in actively SF galaxies. Geach et al. (2006) find an excess of mid-IR sources in an unvirialized cluster at $z \sim 0.4$, where star formation might be triggered via mergers or interactions between gas-rich spirals. However, they also note that significant cluster-to-cluster variations are possible: they do not find any significant excess in another cluster at similar redshift, of similar mass but with a hotter and smoother ICM. Moving to higher redshift, Marcillac et al. (2007) studied $24 \mu \mathrm{m}$ sources in a massive, dynamically young, unvirialized cluster at $z=0.83$. They find that IR-detected galaxies tend to lie in the outskirts of the cluster, while they avoid the merging region. Finally, Elbaz et al. (2007), utilizing
$24 \mu \mathrm{m}$ imaging in the GOODS fields at redshift $0.8<z<1.2$, have identified for the first time a reversal of the SFR-density relation observed at lower redshifts. This result has been recently confirmed by Cooper et al. (2008) with a spectroscopic analysis using DEEP2 data.

\subsection{Dusty or Old?}

The relative abundance of red SF galaxies at intermediate and high densities suggests that they are transforming under the influence of some environment-related process. What are the star formation activity, morphology, and dust attenuation of these red SF galaxies?

1. The red SF galaxies in our sample are not in a starburst phase. The few starburst galaxies (with $\log \left(\mathrm{SFR} / M_{*}\right)>$ -9.7, corresponding to a birthrate parameter $b>1$, assuming a formation redshift of 4) in our sample all populate the blue cloud. We find that red SF galaxies have similar SFR as blue SF galaxies, and slightly lower specific SFR. While the overall fraction of SF galaxies decreases with density, we do not identify any significant evolution in their level of activity, either obscured or not.

2. The morphology of SF galaxies is not very sensitive to their color. Red SF have similar distribution in the Sersic index as blue SF: they are predominantly disk-dominated. Moreover, the morphology of SF galaxies depends little on the environment in which they live. This suggests that, on average, changes in stellar populations and changes in morphology happen on different timescales, as hinted at by the fact that color seems to be more sensitive to environment than morphology (Blanton et al. 2005). The rise of red massive spirals in the infalling regions of the A901/902 cluster has also been interpreted by Wolf et al. (2008) as due to SFR decline not accompanied by morphological change. A two-step scenario in which star formation is quenched first and morphological transformation follows on longer timescale is also supported by the analysis of Sánchez et al. (2007) of the A2218 cluster at $z=0.17$.

3. Red SF galaxies have IR-to-UV luminosity ratios $\left(L_{\mathrm{IR}} / L_{\mathrm{UV}}\right)$, a proxy for the level of UV attenuation by dust, on average higher than blue SF galaxies. The distribution in their IR-to-UV luminosity ratios suggests, however, the presence of two different populations, hence possibly two distinct mechanisms affecting the star formation activity of red galaxies. Roughly half of the red SF galaxies in our sample have relatively low $L_{\mathrm{IR}} / L_{\mathrm{UV}}$, similar to the average value of the bulk of blue SF galaxies, without evolution with environment. The other half of the red SF galaxies have instead systematically higher $L_{\mathrm{IR}} / L_{\mathrm{UV}}$. The range in dust attenuation of this second population becomes narrower at higher densities, suggesting a trend of decreasing attenuation with density.

On the basis of the IR properties of red SF galaxies we tentatively distinguish them into two subpopulations. Lowattenuation red SF galaxies have low specific SFR $\left(\lesssim 10^{-10.3}\right)$ independent of environment. Among SF galaxies they tend to have higher Sersic indices $(\langle n\rangle \sim 2.5)$. These properties suggest that these galaxies are dominated by rather old stellar populations but have some residual star formation. They could be anemic/gas-deficient spirals gradually suppressing their star formation as a consequence of the removal of their gas reservoir as they move into higher-density environments (Fumagalli \& Gavazzi 2008). Their star formation could be suppressed on 
relatively long timescales (of few Gyr) if strangulation of the hot, diffuse gas occurs while the galaxies enter a more massive halo (e.g., Balogh \& Morris 2000; van den Bosch et al. 2008). The gradual fading of the disk would make the morphology of these galaxies appear of earlier type. In addition to strangulation, when the density of the surrounding medium becomes sufficiently high, ram-pressure can act on smaller mass galaxies to remove the remaining gas on the disk and lead to fast quenching (e.g., Gunn \& Gott 1972; Quilis et al. 2000; Boselli et al. 2006). However, there is no significant evidence that the relative abundance of low-attenuation red SF galaxies varies with environment. Therefore, we cannot exclude that these galaxies are suppressing their SF due to internal feedback processes only, without any additional environmental action required.

The other subpopulation of red SF galaxies have systematically higher $L_{\mathrm{IR}} / L_{\mathrm{UV}}$, indicative of higher levels of dust attenuation. They represent $\gtrsim 40 \%$ of all red SF galaxies in the sample, even after accounting for purely edge-on spirals. By visual inspection of their $H S T V$-band images, we can say that the majority of them are spiral galaxies with a bright nucleus or inner bar/disk, suggesting intense star formation activity in the galaxy core (we cannot exclude AGN contribution in some cases). We also find few cases of interacting galaxies and merger remnants. In comparison to the low-attenuation red SF class discussed above, they have systematically higher specific SFR and lower values of the Sersic index $(\langle n\rangle \sim 1.5)$. As opposed to low-attenuation red SF galaxies, they tend to be more abundant at intermediate densities where their stellar mass is $\sim 50 \%$ and $\sim 70 \%$ higher than at low and high densities, respectively. This suggests that environmental interactions are particularly efficient in triggering episodes of obscured, often centrally concentrated, star formation in these massive late-type spirals. While we find few cases of interacting galaxies, violent processes, such as mergers, leading to intense starbursts cannot be the dominant phenomenon. These galaxies are likely more sensitive to more gentle mechanisms that perturb the distribution of gas inducing star formation (but not a starburst) and at the same time increase the gas/dust column density. This process should not alter morphology as long as star formation is still detectable. The fact that galaxies undergoing this phase are preferentially found at intermediate densities and with relatively high stellar masses might indicate longer duration of the dust-obscured episode of SF for more massive galaxies, which are thus more likely to be caught in this phase than low-mass galaxies. Harassment can act on massive spirals, funneling the gas toward the center and leading to a temporary enhancement of star formation (Moore et al. 1998; Lake et al. 1998). The timescales of this process could be relatively long if it occurs at group-like densities, rather than in the cluster. Tidal interactions between galaxies at low and intermediate densities can also produce gas funneling toward the center (Mihos 2004).

In summary, we have identified a significant amount of star formation "hidden" among red-sequence galaxies, contributing at least $30 \%$ to the total star formation activity at intermediate and high densities. The red SF population is composed partly of disk galaxies dominated by old stellar populations and with low-level residual star formation, and partly of spirals or irregular galaxies undergoing modest (non-starburst) episodes of dust-obscured star formation. This means that, while we confirm the general suppression of star formation with increasing environmental density, the small amount of star formation surviving the cluster happens to a large extent in galaxies either obscured or dominated by old stellar populations. Low-attenuation red SF galaxies seem to be a ubiquitous population at all densities. Therefore, an environmental action is not necessarily required to explain their ongoing lowlevel star formation. In contrast, dusty SF galaxies are relatively more abundant at intermediate densities. They might be experiencing harassment or tidal interactions with other galaxies, which funnel gas toward the center inducing a (partly or totally obscured) episode of star formation. Ram-pressure can also be partly responsible for the population of relatively more massive dusty SF galaxies in the cluster: while it is not effective in removing the gas from the disk in massive galaxies, it could perturb it inducing obscured star formation. The complex dynamical state of the A901/902 supercluster could favor a combination of different processes producing a temporary enhancement of obscured star formation.

A.G. thanks Stéphane Charlot for comments on an early draft and Stefano Zibetti for useful discussions. A.G., E.F.B., A.R.R., and K.J. acknowledge support from the Deutsche Forschungsgemeinschaft through the Emmy Noether Programme, C.W. from a PPARC Advanced Fellowship, M.E.G. from an Anne McLaren Research Fellowship, M.B. and E.v.K. by the Austrian Science Foundation F.W.F. under grant P18416. C.Y.P. is grateful for support provided through STScI and NRC-HIA Fellowship programmes. C.H. acknowledges the support of a European Commission Programme Sixth Framework Marie Curie Outgoing International Fellowship under contract MOIF-CT-200621891, and a CITA National Fellowship. D.H.M. acknowledges support from the National Aeronautics and Space Administration (NASA) under LTSA Grant NAG5-13102 issued through the Office of Space Science. A.B. was supported by the DLR (50 OR 0404), S.J. by NASA under LTSA Grant NAG5-13063 and NSF under AST-0607748, S.F.S. by the Spanish MEC grants AYA2005-09413-C02-02 and the PAI of the Junta de Andalucía as research group FQM322. Support for STAGES was provided by NASA through GO-10395 from STScI operated by AURA under NAS5-26555.

\section{REFERENCES}

Alexander, D. M., et al. 2003, AJ, 126, 539

Baldry, I. K., Balogh, M. L., Bower, R. G., Glazebrook, K., Nichol, R. C., Bamford, S. P., \& Budavari, T. 2006, MNRAS, 373, 469

Balogh, M. L., Baldry, I. K., Nichol, R., Miller, C., Bower, R., \& Glazebrook, K. 2004a, ApJ, 615, L101

Balogh, M. L., \& Morris, S. L. 2000, MNRAS, 318, 703

Balogh, M. L., Schade, D., Morris, S. L., Yee, H. K. C., Carlberg, R. G., \& Ellingson, E. 1998, ApJ, 504, L75

Balogh, M., et al. 2004b, MNRAS, 348, 1355

Barnes, J. E. 1988, ApJ, 331, 699

Bekki, K., Shioya, Y., \& Couch, W. J. 2001, ApJ, 547, L17

Bell, E. F. 2003, ApJ, 586, 794

Bell, E. F., McIntosh, D. H., Katz, N., \& Weinberg, M. D. 2003, ApJS, 149, 289 Bell, E. F., Zheng, X. Z., Papovich, C., Borch, A., Wolf, C., \& Meisenheimer, K. 2007, ApJ, 663, 834

Bell, E. F., et al. 2004, ApJ, 608, 752

Bell, E. F., et al. 2005, ApJ, 625, 23

Bertin, E., \& Arnouts, S. 1996, A\&AS, 117, 393

Best, P. N. 2004, MNRAS, 351, 70

Blanton, M. R., Eisenstein, D., Hogg, D. W., Schlegel, D. J., \& Brinkmann, J. 2005, ApJ, 629, 143

Borch, A., et al. 2006, A\&A, 453, 869

Boselli, A., Boissier, S., Cortese, L., Gil de Paz, A., Seibert, M., Madore, B. F., Buat, V., \& Martin, D. C. 2006, ApJ, 651, 811

Boselli, A., \& Gavazzi, G. 2006, PASP, 118, 517

Bressan, A., et al. 2007, in IAU Symp. 241, ed. A. Vazdekis \& R. F. Peletier (Cambridge: Cambridge Univ. Press), 395 
Caldwell, J. A. R., et al. 2008, ApJS, 174, 136

Calzetti, D., et al. 2007, ApJ, 666, 870

Cassata, P., et al. 2007, ApJS, 172, 270

Cassata, P., et al. 2008, A\&A, 483, L39

Cayatte, V., van Gorkom, J. H., Balkowski, C., \& Kotanyi, C. 1990, AJ, 100, 604

Chabrier, G. 2003, ApJ, 586, L133

Coia, D., et al. 2005, A\&A, 431, 433

Cooper, M. C., Newman, J. A., Madgwick, D. S., Gerke, B. F., Yan, R., \& Davis, M. 2005, ApJ, 634, 833

Cooper, M. C., et al. 2008, MNRAS, 383, 1058

Cortese, L., Boselli, A., Franzetti, P., Decarli, R., Gavazzi, G., Boissier, S., \& Buat, V. 2008, MNRAS, 386, 1157

Cortese, L., et al. 2007, MNRAS, 376, 157

Dale, D. A., \& Helou, G. 2002, ApJ, 576, 159

Devriendt, J. E. G., Guiderdoni, B., \& Sadat, R. 1999, A\&A, 350, 381

Dressler, A. 1980, ApJ, 236, 351

Dressler, A., et al. 1997, ApJ, 490, 577

Duc, P.-A., et al. 2002, A\&A, 382, 60

Elbaz, D., et al. 2007, A\&A, 468, 33

Fadda, D., Biviano, A., Marleau, F. R., Storrie-Lombardi, L. J., \& Durret, F. 2008, ApJ, 672, L9

Fadda, D., Elbaz, D., Duc, P.-A., Flores, H., Franceschini, A., Cesarsky, C. J., \& Moorwood, A. F. M. 2000, A\&A, 361, 827

Franzetti, P., et al. 2007, A\&A, 465, 711

Fujita, Y. 2004, PASJ, 56, 29

Fumagalli, M., \& Gavazzi, G. 2008, A\&A, 490, 571

Gavazzi, G., Boselli, A., Cortese, L., Arosio, I., Gallazzi, A., Pedotti, P., \& Carrasco, L. 2006, A\&A, 446, 839

Gavazzi, G., Boselli, A., Pedotti, P., Gallazzi, A., \& Carrasco, L. 2002, A\&A, 396, 449

Gavazzi, G., Cortese, L., Boselli, A., Iglesias-Paramo, J., Vílchez, J. M., \& Carrasco, L. 2003, ApJ, 597, 210

Gavazzi, G., \& Jaffe, W. 1985, ApJ, 294, L89

Geach, J. E., et al. 2006, ApJ, 649, 661

Gilmour, R., Gray, M. E., Almaini, O., Best, P., Wolf, C., Meisenheimer, K., Papovich, C., \& Bell, E. 2007, MNRAS, 380, 1467

Giovanelli, R., \& Haynes, M. P. 1985, ApJ, 292, 404

Gómez, P. L., et al. 2003, ApJ, 584, 210

Gordon, K. D., Clayton, G. C., Witt, A. N., \& Misselt, K. A. 2000, ApJ, 533, 236

Goto, T., et al. 2003, PASJ, 55, 757

Gray, M. E., Taylor, A. N., Meisenheimer, K., Dye, S., Wolf, C., \& Thommes, E. 2002, ApJ, 568, 141

Gray, M. E., Wolf, C., Meisenheimer, K., Taylor, A., Dye, S., Borch, A., \& Kleinheinrich, M. 2004, MNRAS, 347, L73

Gray, M. E., et al. 2008, MNRAS, in press, arXiv:0811.3890

Gunn, J. E., \& Gott, J. R. I. 1972, ApJ, 176, 1

Hammer, F., et al. 1997, ApJ, 481, 49

Helou, G., Khan, I. R., Malek, L., \& Boehmer, L. 1988, ApJS, 68, 151

Heymans, C., et al. 2005, MNRAS, 361, 160

Heymans, C., et al. 2008, MNRAS, 385, 1431

Jain, B., \& Van Waerbeke, L. 2000, ApJ, 530, L1

Kauffmann, G., White, S. D. M., \& Guiderdoni, B. 1993, MNRAS, 264, 201

Kauffmann, G., White, S. D. M., Heckman, T. M., Ménard, B., Brinchmann, J., Charlot, S., Tremonti, C., \& Brinkmann, J. 2004, MNRAS, 353, 713

Kaviraj, S., et al. 2007, ApJS, 173, 619

Kennicutt, R. C., Jr. 1983, AJ, 88, 483

Kennicutt, R. C., Jr. 1998, ARA\&A, 36, 189

Kennicutt, R. C., Roettiger, K. A., Keel, W. C., van der Hulst, J. M., \& Hummel, E. 1987, AJ, 93, 1011

Kinney, A. L., Calzetti, D., Bohlin, R. C., McQuade, K., Storchi-Bergmann, T., \& Schmitt, H. R. 1996, ApJ, 467, 38

Koopmann, R. A., \& Kenney, J. D. P. 2004, ApJ, 613, 866

Kroupa, P. 2001, MNRAS, 322, 231

Kroupa, P., Tout, C. A., \& Gilmore, G. 1993, MNRAS, 262, 545

Lake, G., Katz, N., \& Moore, B. 1998, ApJ, 495, 152

Larson, R. B., Tinsley, B. M., \& Caldwell, C. N. 1980, ApJ, 237, 692

Lewis, I., et al. 2002, MNRAS, 334, 673

Marcillac, D., Rigby, J. R., Rieke, G. H., \& Kelly, D. M. 2007, ApJ, 654, 825
McIntosh, D. H., Rix, H.-W., \& Caldwell, N. 2004, ApJ, 610, 161

Mihos, J. C. 2004, in Clusters of Galaxies: Probes of Cosmological Structure and Galaxy Evolution, ed. J. S. Mulchaey, A. Dressler, \& A. Oemler (Cambridge: Cambridge Univ. Press), 277

Miller, N. A., \& Owen, F. N. 2002, AJ, 124, 2453

Miller, N. A., \& Owen, F. N. 2003, AJ, 125, 2427

Miyazaki, S., et al. 2002, ApJ, 580, L97

Moore, B., Lake, G., \& Katz, N. 1998, ApJ, 495, 139

Moran, S. M., Ellis, R. S., Treu, T., Smith, G. P., Rich, R. M., \& Smail, I. 2007, ApJ, 671, 1503

Morgan, W. W. 1961, Proc. Natl Acad. Sci., 47, 905

Moss, C. 2006, MNRAS, 373, 167

Oemler, A. J. 1974, ApJ, 194,

Papovich, C., \& Bell, E. F. 2002, ApJ, 579, L1

Papovich, C., et al. 2004, ApJS, 154, 70

Peng, C. Y., Ho, L. C., Impey, C. D., \& Rix, H.-W. 2002, AJ, 124, 266

Pimbblet, K. A., Smail, I., Kodama, T., Couch, W. J., Edge, A. C., Zabludoff, A. I., \& O’Hely, E. 2002, MNRAS, 331, 333

Poggianti, B. M., Smail, I., Dressler, A., Couch, W. J., Barger, A. J., Butcher, H., Ellis, R. S., \& Oemler, A. J. 1999, ApJ, 518, 576

Porter, S. C., Raychaudhury, S., Pimbblet, K. A., \& Drinkwater, M. J. 2008 MNRAS, 388, 1152

Postman, M., et al. 2005, ApJ, 623, 721

Quilis, V., Moore, B., \& Bower, R. 2000, Science, 288, 1617

Ramos Almeida, C., Pérez García, A. M., Acosta-Pulido, J. A., \& Rodríguez Espinosa, J. M. 2007, AJ, 134, 2006

Relaño, M., Lisenfeld, U., Pérez-González, P. G., Vílchez, J. M., \& Battaner, E. 2007, ApJ, 667, L141

Rieke, G. H., et al. 2004, ApJS, 154, 25

Risaliti, G., Maiolino, R., \& Salvati, M. 1999, ApJ, 522, 157

Rix, H.-W., et al. 2004, ApJS, 152, 163

Rogers, B., Ferreras, I., Lahav, O., Bernardi, M., Kaviraj, S., \& Yi, S. K. 2007, MNRAS, 382, 750

Rowan-Robinson, M., et al. 2005, AJ, 129, 1183

Saintonge, A., Tran, K.-V.-H., \& Holden, B. P. 2008, ApJ, 685, L113

Sánchez, S. F., Cardiel, N., Verheijen, M. A. W., Pedraz, S., \& Covone, G. 2007 MNRAS, 376, 125

Smail, I., Morrison, G., Gray, M. E., Owen, F. N., Ivison, R. J., Kneib, J.-P., \& Ellis, R. S. 1999, ApJ, 525, 609

Smith, G. P., Treu, T., Ellis, R. S., Moran, S. M., \& Dressler, A. 2005, ApJ, 620 78

Solanes, J. M., Manrique, A., García-Gómez, C., González-Casado, G., Giovanelli, R., \& Haynes, M. P. 2001, ApJ, 548, 97

Springel, V., Di Matteo, T., \& Hernquist, L. 2005, MNRAS, 361, 776

Taylor, A. N., et al. 2004, MNRAS, 353, 1176

Temi, P., Brighenti, F., \& Mathews, W. G. 2005, ApJ, 635, L25

Temi, P., Brighenti, F., \& Mathews, W. G. 2007, ApJ, 660, 1215

Temi, P., Brighenti, F., \& Mathews, W. G. 2008, ApJ, 672, 244

Toomre, A., \& Toomre, J. 1972, ApJ, 178, 623

Treu, T., Ellis, R. S., Kneib, J.-P., Dressler, A., Smail, I., Czoske, O., Oemler, A., \& Natarajan, P. 2003, ApJ, 591, 53

van den Bergh, S. 1976, ApJ, 206, 883

van den Bosch, F. C., Aquino, D., Yang, X., Mo, H. J., Pasquali, A., McIntosh, D. H., Weinmann, S. M., \& Kang, X. 2008, MNRAS, 387, 79

van der Wel, A. 2008, ApJ, 675, L13

van Waerbeke, L. 2000, MNRAS, 313, 524

Verdugo, M., Ziegler, B. L., \& Gerken, B. 2008, A\&A, 486, 9

Wilman, D. J., et al. 2008, ApJ, 680, 1009

Wolf, C., Gray, M. E., Aragón-Salamanca, A., Lane, K. P., \& Meisenheimer, K. 2007, MNRAS, 376, L1

Wolf, C., Gray, M. E., \& Meisenheimer, K. 2005, A\&A, 443, 435

Wolf, C., Meisenheimer, K., Rix, H.-W., Borch, A., Dye, S., \& Kleinheinrich, M. 2003, A\&A, 401, 73

Wolf, C., et al. 2004, A\&A, 421, 913

Wolf, C., et al. 2008, MNRAS, in press, arXiv:0811.3873

Yi, S. K., et al. 2005, ApJ, 619, L111

Young, L. M., Bendo, G. J., \& Lucero, D. M. 2008, AJ, accepted, arXiv:0811.1381

Zabludoff, A. 2002, in AMiBA 2001: High-Z Clusters, Missing Baryons, and CMB Polarization, ASP Conf. Proc. 257, ed. L.-W. Chen, et al. (San Francisco, CA: ASP), 123 